This article has been accepted for publication in Monthly Notices of the Royal Astronomical Society (C): 2020 The Authors. Published by Oxford University Press on behalf of the Royal Astronomical Society. All rights reserved. 


\title{
Morphology of radio relics - I. What causes the substructure of synchrotron emission?
}

\author{
P. Domínguez-Fernández ${ }^{\circledR},{ }^{\star}{ }^{\star}$ M. Brüggen ${ }^{\circledR},{ }^{1}$ F. Vazza ${ }^{\circledR},{ }^{1,2,3}$ W. E. Banda-Barragán ${ }^{\circledR},{ }^{1}$ \\ K. Rajpurohit ${ }^{\circledR},{ }^{2,3}$ A. Mignone ${ }^{\circledR},{ }^{4}$ D. Mukherjee ${ }^{\circledR 5}$ and B. Vaidya ${ }^{\circledR 6}$ \\ ${ }^{1}$ Hamburger Sternwarte, Gojenbergsweg 112, D-21029 Hamburg, Germany \\ ${ }^{2}$ Dipartimento di Fisica e Astronomia, Universitá di Bologna, Via Gobetti 92/3, I-40121 Bologna, Italy \\ ${ }^{3}$ Istituto di Radio Astronomia, INAF, Via Gobetti 101, I-40121 Bologna, Italy \\ ${ }^{4}$ Dipartimento di Fisica, Università di Torino, via Pietro Giuria 1, I-10125 Torino, Italy \\ ${ }^{5}$ Inter-University Centre for Astronomy and Astrophysics, Post Bag 4, Pune 411007, India \\ ${ }^{6}$ Discipline of Astronomy, Astrophysics and Space Engineering, Indian Institute of Technology Indore, Khandwa Road, Simrol, Indore 453552, India
}

Accepted 2020 September 27. Received 2020 September 18; in original form 2020 August 17

\begin{abstract}
High-resolution radio observations of cluster radio relics often show complex spatial and spectral features. However, it is not clear what these features reveal about the underlying magnetic field properties. We performed three-dimensional magnetohydrodynamical simulations of merger shock waves propagating through a magnetized, turbulent intracluster medium. Our model includes the diffusive shock acceleration (DSA) of cosmic ray electrons, their spatial advection and energy losses at run-time. With this set-up we can investigate the relation between radio substructure and pre-shock plasma conditions in the host cluster. We find that upstream turbulence plays a major role in shaping the properties of radio relics produced downstream. Within the assumption of DSA, we can reproduce the observed discrepancy between the X-ray derived Mach number of shocks, and the Mach number inferred from radio spectra. Our simulated spectral index maps and profiles across the radio relic also suggest that the standard deviation of the upstream magnetic field must be relatively small $\left(\sigma_{B} \leq 1 \mu \mathrm{G}\right)$ in order to reproduce observations and therefore radio relics can potentially constrain the distribution of magnetic fields in galaxy clusters outskirts.
\end{abstract}

Key words: acceleration of particles - shock waves - methods: numerical-galaxies: clusters: intracluster medium.

\section{INTRODUCTION}

Radio observations of galaxy clusters reveal non-thermal processes in the intracluster medium (ICM). The size of diffuse radio sources is of the order of Mpc. Such large-scale emission is typically grouped into two categories: radio haloes and radio relics. The first category refers to objects located at the cluster centre and of a relatively regular and spherical shape, with little to absent polarization (e.g. Brunetti $\&$ Jones 2014). The second category, refers to objects located at the cluster periphery, with elongated shapes and typically large degrees of polarization (see Brüggen et al. 2012; van Weeren et al. 2019 for reviews). Radio relics are the main focus of this work.

The particle acceleration mechanisms causing diffuse radio sources are not fully understood. However, it seems clear that shocks generated during the assembly of galaxy clusters play a key role in accelerating the synchrotron-emitting cosmic ray (CR) electrons (see van Weeren et al. 2011 or Bykov et al. 2019 for a review). The radio emission observed in relics is compatible with synchrotron emission from CR electrons with Lorentz factors of $\gamma_{e} \sim 10^{3}-10^{5}$, which are believed to be accelerated via mild shocks $\left(\mathcal{M}_{\text {radio }}{ }^{1} \sim 1.7-4.6\right)$

\footnotetext{
^ E-mail: pdominguez@hs.uni-hamburg.de

${ }^{1} \mathcal{M}_{\text {radio }}$ is the Mach number inferred from radio observations.
}

crossing the ICM (e.g. Clarke \& Ensslin 2006; van Weeren et al. 2010, 2012).

The first-order Fermi acceleration process, commonly known as diffusive shock acceleration (DSA), explains the acceleration of relativistic particles by the passage of a collisionless shock wave (e.g. Blandford \& Ostriker 1978; Drury 1983). A small fraction $\left(\ll 10^{-3}\right)$ of thermal particles can cross the shock front multiple times, and receive a boost in their momentum proportional to $\Delta v / c$, where $\Delta v$ is the velocity jump (difference) across the shock. This acceleration mechanism is observed to be much more efficient than what is expected from theory (see van Weeren et al. 2019; Botteon et al. 2020). Hence, it has been proposed that the electrons are preaccelerated (e.g. Kang, Ryu \& Jones 2012; Pinzke, Oh \& Pfrommer 2013) before they enter the DSA mechanism.

Moreover, the DSA process does not offer a straightforward explanation for the non-detection of $\gamma$-rays from clusters (see Ackermann et al. 2010, 2014, 2016; Vazza \& Brüggen 2014). Among the most relevant open challenges in our understanding of radio relics are (i) the discrepancy between the Mach numbers detected in Xrays and those inferred from radio observations assuming DSA (e.g. Botteon et al. 2020), and (ii) the high electron acceleration efficiency of the order of several percent for the weak shocks commonly associated with radio relics. Up until now, only a few radio relics can readily be explained by the DSA model (e.g. Locatelli et al. 2020). 
Recent high-resolution radio observations have shown a plethora of complex structures in radio relics (e.g. Owen et al. 2014; van Weeren et al. 2017; Di Gennaro et al. 2018; Rajpurohit et al. 2018, 2020). Attempts to explain the observed features struggle with the vast range of scales, from cosmological scales ( $\gtrsim \mathrm{Mpc}$ ), down to turbulent scales $(\sim 10 \mathrm{kpc})$ (e.g. Egan et al. 2016; DomínguezFernández et al. 2019), or even down to plasma scales where particle acceleration occurs $\left(\sim 10^{-6} \mathrm{kpc}\right.$ for the largest gyroradius of relativistic protons). A possible choice is to study particle acceleration from the microphysical point of view using Particle In Cell (PIC) simulations (e.g. Caprioli \& Spitkovsky 2014a,b; Guo, Sironi \& Narayan 2014; Park, Caprioli \& Spitkovsky 2015; Kang, Ryu \& Ha 2019; Ryu, Kang \& Ha 2019), or conversely on larger scales using the magnetohydrodynamical (MHD) approximation, as customarily done with cosmological simulations (the reader may refer to Donnert et al. 2018 for a review).

Radio emission from radio relics has been modelled in previous works on larger scales (e.g. Skillman et al. 2013; Hong, Kang \& Ryu 2015; Nuza et al. 2017). Due to the discrepancy in scales, it is not possible for MHD simulations to model the emission produced by single electrons, but rather from an ensemble of tracer particles, representing a whole distribution of electrons. Previously, this approach has been applied to cosmological MHD simulations in post-processing (see Wittor et al. 2019).

In this paper, we model the synchrotron emission at run-time in a small fraction of the ICM by means of a new hybrid particle and fluid framework using the MHD code PLUTO (Mignone et al. 2007; Vaidya et al. 2018). Our aim is to study the substructure observed in radio relics (e.g. Rajpurohit et al. 2020). This method uses Lagrangian particles embedded in a large-scale MHD flow, each with its individual energy spectrum. Here, we consider a simplified scenario: we set up a shock tube in a turbulent medium that is representative of a small region of the ICM. We then assume that $\mathrm{CR}$ electrons are injected instantly at the shock discontinuity and acquire spectral properties according to DSA theory.

The paper is structured as follows: In Section 2, we describe our numerical set-up and initial conditions. In Section 3, we include a description of the particles' initial spectral distribution and evolution. In Section 3.3, we explain how we obtain the emission and spectral index maps. Section 4 shows our results and we summarize in Section 5 .

\section{NUMERICAL SET-UP}

\subsection{Initial conditions: modelling the turbulent ICM with FLASH}

The turbulent ICM initial conditions were produced using the MHD FLASH code version 4.6.1 (Fryxell et al. 2000; Calder et al. 2002), with the goal of generating realistic pre-shock conditions. We used the unsplit staggered mesh MHD solver which uses a constrained transport (CT) method at cell interfaces for preserving the divergence-free magnetic field property on a staggered grid (e.g. Lee, Deane \& Federrath 2009). The simulation domain is chosen to be a cubic box of size $L=L_{x}=L_{y}=L_{z}=200 \mathrm{kpc}$, uniformly spaced over a $256^{3}$ cells grid, with periodic boundary conditions. We assumed an ideal gas equation of state (EOS) with $\gamma_{0}=5 / 3$.

Turbulence was created following a spectral forcing method, based on the stochastic Ornstein-Uhlenbeck (OU) process to model the turbulent acceleration $f$ with a finite autocorrelation time (e.g. Eswaran \& Pope 1988; Schmidt, Niemeyer \& Hillebrandt 2006; Federrath et al. 2010). The OU process describes the evolution of the forcing term in Fourier space, $\hat{f}$, with a stochastic differential equation:

$\mathrm{d} \hat{\boldsymbol{f}}(\boldsymbol{k}, t)=f_{0}(\boldsymbol{k}) \mathcal{P}^{\zeta}(\boldsymbol{k}) \mathrm{d} \boldsymbol{W}(t)-\hat{\boldsymbol{f}}(\boldsymbol{k}, t) \frac{\mathrm{d} t}{T}$,

where $f_{0}$ is the forcing amplitude, $\boldsymbol{W}(t)$ is a random Wiener process, $\mathcal{P}^{\zeta}$ is a projection tensor in Fourier space, $\zeta$ is the forcing parameter $[\zeta=0(1)$ for purely compressive(solenoidal) forcing], and $T$ is the autocorrelation time-scale of the forcing (the reader may refer to Federrath et al. 2010 for a detailed explanation on turbulence forcing). In this work, we solely focus on solenoidal subsonic turbulence forcing $(\nabla \cdot f=0)$, since several authors have shown that the most dominant type of turbulence in the ICM should be subsonic with a large ( $\geq 70$ per cent) predominance of solenoidal modes (e.g. Miniati 2014; Vazza et al. 2017).

We have run two simulations with two different stirring scales. The forcing amplitude, $f_{0}$, was set to be a paraboloid in Fourier space in both simulations only containing power on the largest scales in a small interval of wavenumbers. We chose two different intervals: $1 \leq k L / 2 \pi \leq 2$ for the first simulation and $1 \leq k L / 2 \pi \leq 4$ for the second simulation. As the power peaks in $2 / 3$ and $1 / 4$ of the box, we will refer to each of them as $2 L / 3$ and $L / 4$, representing injection scales of 133 and $50 \mathrm{kpc}$, respectively. Furthermore, the maximum $k$ allowed in the box corresponds to the $2 L / 3$ case. Conversely, the $L / 4$ case satisfies the minimum condition where its eddy turnover time is smaller than the time needed for our main set-up (where a shock sweeps this turbulent medium).

The autocorrelation time-scale was set equal to the dynamical time-scale (also called eddy turn-over time-scale) on the scale of energy injection, $t_{2 L / 3}=2 L / 3 \sigma_{v}$ and $t_{L / 4}=L / 4 \sigma_{v}$, respectively, where $\sigma_{v}$ is the rms velocity to be achieved at saturation. Both simulations were set to have an amplitude of the fluctuations of $\sigma_{v}=125 \mathrm{~km} \mathrm{~s}^{-1}$. The turbulence is fully developed after roughly two dynamical timescales when the magnetic energy, the plasma beta, and the rms Mach number become stable (although some transient fluctuations can still be present depending on the balance between mechanical energy from the forcing term and the dissipation rate). At this point, the magnetic field reaches a saturated state since we start with a relatively strong magnetic field seed of $0.2 \mu \mathrm{G}$ (for the $2 L / 3$ case) and $0.4 \mu \mathrm{G}$ (for the $L / 4$ case). This is shown in Fig. 1 where we plot the evolution of the total kinetic and magnetic energy and in Fig. 2, where we show the evolution of the rms Mach number and the plasma beta, $\beta$, for both runs. Even after the magnetic saturation, the thermal pressure fluctuations will continue to increase due to turbulent dissipation and, as a consequence, the rms sonic Mach number decreases.

We selected one snapshot from each of these two runs to represent a small region of the ICM and each act as an initial condition (see Section 2 and Fig. 5). The snapshots are taken at the respective saturation times, which is $t=2.1 \mathrm{Gyr}$ for the run with injection scale $2 L / 3$ and $t=0.78 \mathrm{Gyr}$ for the run with injection scale $L / 4$. In Fig. 3, we show the probability distribution function of the magnetic field strength at those times. The two snapshots have a sufficiently different distribution of magnetic fields (due to the difference in the initial magnetic field seed and the interval of wavenumbers of the stirring), yet in terms of the rms sonic Mach number and plasma- $\beta$ they lie in the range of characteristic values of the ICM (e.g. Ryu et al. 2008). Note that the tail of the $L / 4$ distribution extends to $\sim 2$ times larger magnetic field values (see Fig. 3). For reference we include the PDFs of one galaxy cluster previously produced in a cosmological MHD simulation and analysed in Vazza et al. (2018) and Domínguez-Fernández et al. (2019). This cluster is classified as 


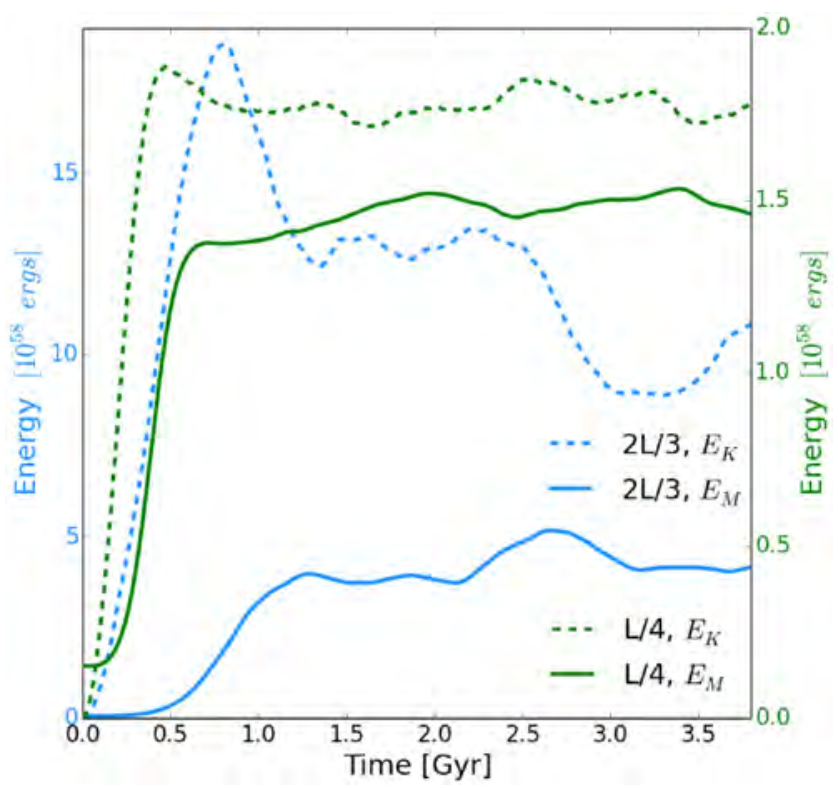

Figure 1. Energy evolution for the runs with injection at $2 L / 3$ (blue) and at $L / 4$ (green). The kinetic energy is shown with dashed lines and the magnetic energy with solid lines. Saturation is reached at $t=2 t_{2 L / 3}=2.1 \mathrm{Gyr}$ and $t=$ $2 t_{L / 4}=0.78 \mathrm{Gyr}$, respectively. After saturation the energy ratio, $E_{M} / E_{K}$, is $\sim 0.5$ and $\sim 0.85$ for the $2 \mathrm{~L} / 3$ and $L / 4$ cases, respectively.

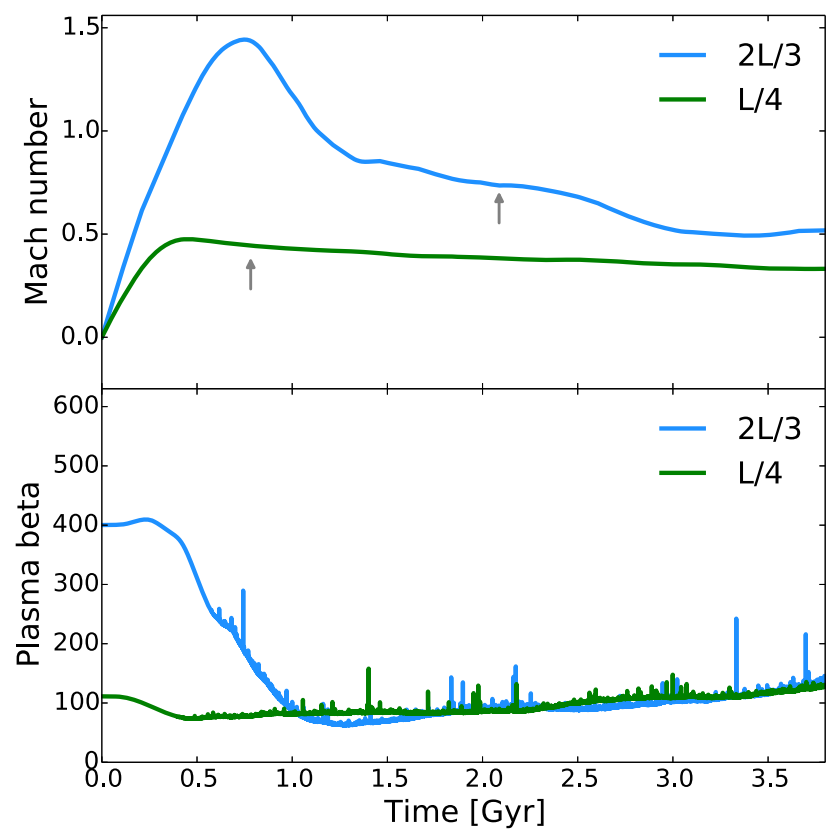

Figure 2. Top panel: Evolution of rms Mach number for the runs with injection at $2 L / 3$ (blue) and at $L / 4$ (green). The arrows point to the selected snapshots to be our initial conditions. Bottom panel: Corresponding evolution of the plasma beta, $\beta$. The selected snapshots have an rms Mach number of $\mathcal{M}_{s} \sim 0.45-0.7$ and a plasma beta of $\beta \sim 110$.

a post-merger $(\mathrm{PM})$ cluster with a mass $M_{200}=1.9 \times 10^{15} \mathrm{M}_{\odot} \cdot{ }^{2}$ We selected a region at a distance of $\sim 1 \mathrm{Mpc}$ from the cluster's

${ }^{2}$ The reader can find this galaxy cluster in Domínguez-Fernández et al. (2019) with ID E16B.

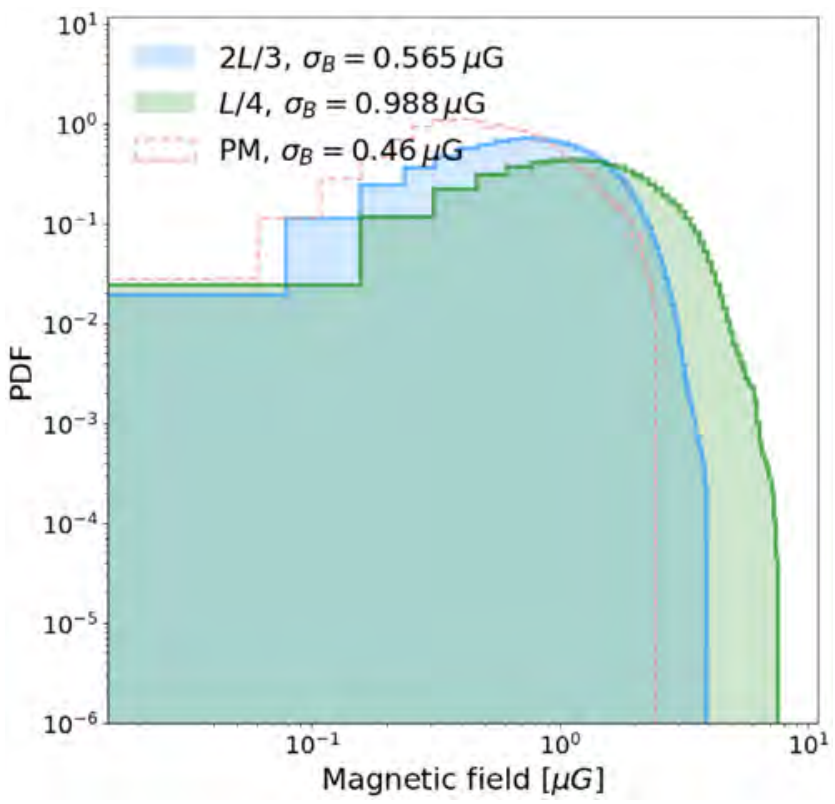

Figure 3. PDFs of magnetic field strength at the selected time for the runs with injection at $2 L / 3$ (blue) and at $L / 4$ (green). Extra dashed line: PDF of a post-merger (PM) galaxy cluster previously analysed in DomínguezFernández et al. (2019).

centre with an extent of $\sim 250 \mathrm{kpc}$. As can be seen in Fig. 3, the PDF of the magnetic field broadly agrees with previous results from cosmological MHD simulations. The high-magnetic field tail of the distribution is slightly more extended than in cosmological simulations, owing to the larger Reynolds number in these new simulations. The magnetic field strength at the outskirts of the clusters is most likely underestimated in the cosmological simulation due to the limited resolution (see Vazza et al. 2018). A more extensive survey of the interaction between merger shocks with a larger range of different initial turbulent conditions for the ICM will be the subject of future work.

\subsection{Main PLUTO simulations}

In order to study the synchrotron emission in an MHD shock tube, we use the code PLUTO (Mignone et al. 2007), which solves the following conservation laws for ideal MHD:

$\frac{\partial \rho}{\partial t}+\nabla \cdot(\rho \mathbf{v})=0$

$\frac{\partial \boldsymbol{m}}{\partial t}+\nabla \cdot\left[\boldsymbol{m} \boldsymbol{v}-\boldsymbol{B} \boldsymbol{B}+\boldsymbol{I}\left(p+\frac{B^{2}}{2}\right)\right]^{T}=0$,

$\frac{\partial\left(E_{t}\right)}{\partial t}+\nabla \cdot\left[\left(\frac{\rho v^{2}}{2}+\rho e+p\right) \boldsymbol{v}-(\boldsymbol{v} \times \boldsymbol{B}) \times \boldsymbol{B}\right]=0$,

$\frac{\partial \boldsymbol{B}}{\partial t}-\nabla \times(\boldsymbol{v} \times \boldsymbol{B})=0$,

$\nabla \cdot \boldsymbol{B}=0$,

$\rho e=\frac{p}{\gamma_{0}-1}$,

where $\rho$ is the gas mass density, $\boldsymbol{m}=\rho \boldsymbol{v}$ is the momentum density, $p$ is the thermal pressure, $\boldsymbol{B}$ is the magnetic field (where a factor of $1 / \sqrt{4 \pi}$ has been absorbed in its definition), $E_{t}$ is the total energy density, $e$ the specific internal energy and where we assumed an ideal EOS, that is $\gamma_{0}=5 / 3$. 


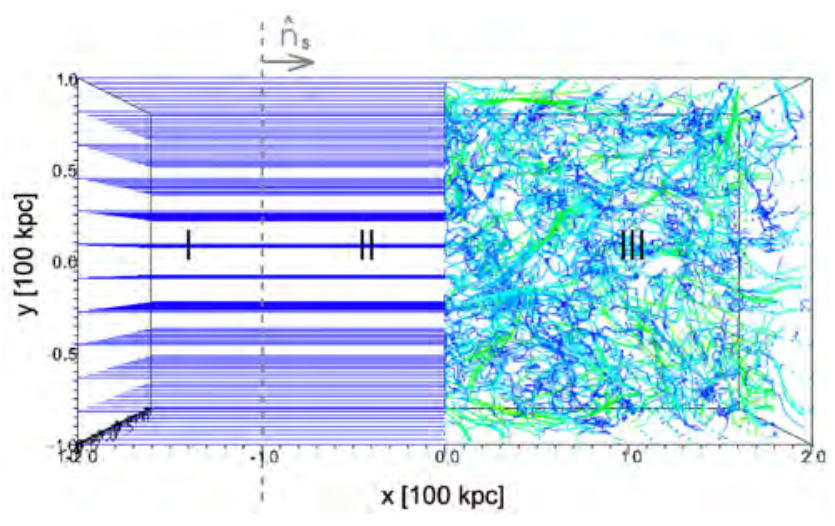

Figure 4. Initial magnetic field configuration in the PLUTO code. The streamlines are coloured according to the magnitude of the magnetic field: green, light colours denote higher values, while dark blue colour indicates lower values. I denotes the post-shock region, II denotes the uniform preshock region, and III the turbulent pre-shock region (see Section 2.1). The left-hand side is a uniform medium with a $B_{x}$ component matching the mean value of the $B_{x}$ of the turbulent medium. We have one Lagrangian particle per cell placed in the whole regions II and III.

Our computational domain is a rectangular box $(400 \mathrm{kpc} \times 200 \mathrm{kpc}$ $\times 200 \mathrm{kpc}$ with $256 \times 128 \times 128$ cells, respectively), where $x \in$ $[-200,200] \mathrm{kpc}, y \in[-100,100] \mathrm{kpc}$, and $z \in[-100,100] \mathrm{kpc}$ (see Fig. 4). The right-hand half of the domain is filled with a turbulent medium (see Section 2.1), representing a realistic ICM, while the left-hand half contains a uniform medium in which the shock is launched. We define a shock discontinuity at $x=-100 \mathrm{kpc}$ (see Fig. 4 for the initial configuration of the magnetic field). This defines three regions in our simulation box: a post-shock uniform region (I), a pre-shock uniform region (II) and a pre-shock turbulent region (III).

The turbulent medium is produced externally, with the procedure outlined in Section 2.1. The turbulent fields are then read into PLUTO and interpolated on to the numerical mesh used to evolve shocks with a bi- or tri-linear interpolation at the desired coordinate location at the initial time.The initial boundary conditions of the computational domain are outflow in $x$ (zero gradient across the boundary) and periodic in $y$ and $z$. We used a piecewise parabolic method for the spatial integration, whereas a second-order TVD RungeKutta method for the time stepping with a Courant-Friedichs-Lewy condition of 0.3 . The Riemann solver for the flux computation that we used is the Harten-Lax-van Leer-Discontinuities (HLLD) solver (see Miyoshi \& Kusano 2005). We control the $\nabla \cdot \boldsymbol{B}=0$ condition with the hyperbolic divergence cleaning technique where the induction equation is coupled to a generalized Lagrange multiplier (GLM, e.g. Dedner et al. 2002). In Appendix A, we compare how the GLM and CT methods control the divergence-free condition and find that both methods work reliably in PLUTO.

The initial conditions for the density, pressure and velocity in region II (pre-shock uniform region at $[-100,0] \mathrm{kpc}$ ) are set to the mean value of the corresponding turbulent fields. In the case of the magnetic field in region II, we set it to be the mean value of the $B_{x}$ component of the turbulent medium. The initial conditions for region I (post-shock region) are selected according to the MHD RankineHugoniot conditions (e.g. Landau \& Lifshitz 1987). We performed simulations with two different turbulent media (see Section 2.1) produced with the code FLASH (e.g. Fryxell et al. 2000; Calder et al. 2002) by varying also the strength of the shock and the angle, $\theta_{\mathrm{bn}}$, of the upstream magnetic field ${ }^{3}$ with respect to the normal of the shock. Shocks can be classified as quasi-parallel and quasi-perpendicular if $\theta_{\mathrm{bn}} \leq 45^{\circ}$ or $\theta_{\mathrm{bn}}>45^{\circ}$, respectively. We consider two limits, i.e. $\theta_{\mathrm{bn}}=0^{\circ}$ and $\theta_{\mathrm{bn}}=90^{\circ}$. This sums up to a total of six runs for which all the parameters are summarized in Table 1 . We show the projection maps of the two different initial turbulent media considered for this work in Fig. 5 and clarify how these were selected in Section 2.1.

Finally, we fill the computational domain from the shock discontinuity up to the right side of the box with one Lagrangian particle per cell. This gives us a total number of 3145728 Lagrangian particles for each run.

\section{NON-THERMAL RADIO EMISSION FROM SHOCKS}

\subsection{Particle energy spectrum}

Each Lagrangian particle represents an ensemble of CR electrons and is characterized by an energy distribution function,

$\chi(E, \tau)=\frac{N(E, \tau)}{n_{0}}$,

which gives the number of electrons per fluid number density. These particles evolve according to the CR transport equation,

$\frac{\mathrm{d} \chi}{\mathrm{d} \tau}+\frac{\partial}{\partial E}\left[\left(-\frac{E}{3} \nabla_{\mu} u^{\mu}-c_{r} E^{2}\right) \chi\right]=0$,

where the first term in square brackets accounts for energy losses due to adiabatic expansion, while the second one accounts for the synchrotron and inverse-Compton losses for electrons with isotropically distributed velocity vectors,

$c_{r}=\frac{4}{3} \frac{\sigma_{T} c \beta^{2}}{m_{e}^{2} c^{4}}\left[\frac{B^{2}}{8 \pi}+a_{\mathrm{rad}} T_{\mathrm{CMB}}^{4}(1+z)^{4}\right]$

where $\beta=v_{e} / c$ is the velocity of the electrons, $m_{e}$ their mass, and $a_{\mathrm{rad}}$ the radiation constant. For this work, we assume a constant redshift of $z=0$. The reader may refer to Vaidya et al. (2018) for a complete description of the numerical implementation and the semi-analytical scheme used for solving equation (9).

We study a simplified scenario where the non-thermal spectra of the particles are activated instantly at the shock discontinuity. While the particles follow the fluid flow since $t=0$, the particle's energy distribution starts to evolve only when the particles have passed a shock. We implemented a shock finder based on converging flows and pressure jumps that we describe in Appendix B.

Once the Lagrangian particles are activated at the shock discontinuity, they get assigned an initial energy distribution. The corresponding particle momentum distribution function is a powerlaw distribution, i.e. $f(p) \propto p^{-q}$. The power-law index is given by the DSA theory (Drury 1983).

$q=\frac{3 r}{r-1}$,

where $r$ is the shock compression ratio defined as the ratio of the upstream and downstream densities. If we consider test particle acceleration at a shock of Mach number $\mathcal{M}$, then it is possible to

${ }^{3}$ Note that here we define the direction of the upstream magnetic field as the direction of the mean magnetic field of the turbulent medium. 
Table 1. Initial conditions: we denote our regions I, II, III where I is the post-shock region ([-2, -1$]$ in box coordinates), II is the uniform pre-shock region ( $[-1,0]$ in box coordinates), and III is the turbulent pre-shock region $([0,2])$. The initial conditions for the left-hand side of the shock (region I) depend on the pre-shock conditions (region II) and the initial Mach number of the shock $\mathcal{M}$ through the Rankine-Hugoniot jump conditions. $L$ denotes the length of the turbulent region, i.e. $200 \mathrm{kpc}$ (see more details in Section 2.1). Note that the magnetic field in region II has only an $x$-component.

\begin{tabular}{lccccc}
\hline Run ID & Turbulent medium & $\mathcal{M}$ & $\left.\theta_{\text {bn }}{ }^{\circ}\right)$ & $\rho_{\text {II }}\left(10^{-27} \mathrm{~g} \mathrm{~cm}^{-3}\right)$ & $B_{x, \mathrm{II}}(\mu \mathrm{G})$ \\
\hline k1p5_M2_parallel & $2 L / 3$ & 2.0 & 0 & 1.338 & 0.4 \\
k1p5_M3_parallel & $2 L / 3$ & 3.0 & 0 & 1.338 & 0.4 \\
k4_M2_parallel & $L / 4$ & 2.0 & 0 & 1.338 & 0.4 \\
k4_M3_parallel & $L / 4$ & 3.0 & 0 & 1.338 & 0.4 \\
k1p5_M3_perpendicular & $2 L / 3$ & 3.0 & 90 & 1.338 & $1.156 \times 10^{-12}$ \\
k4_M3_perpendicular & $L / 4$ & 3.0 & 90 & 1.338 & $1.156 \times 10^{-12}$ \\
\hline
\end{tabular}

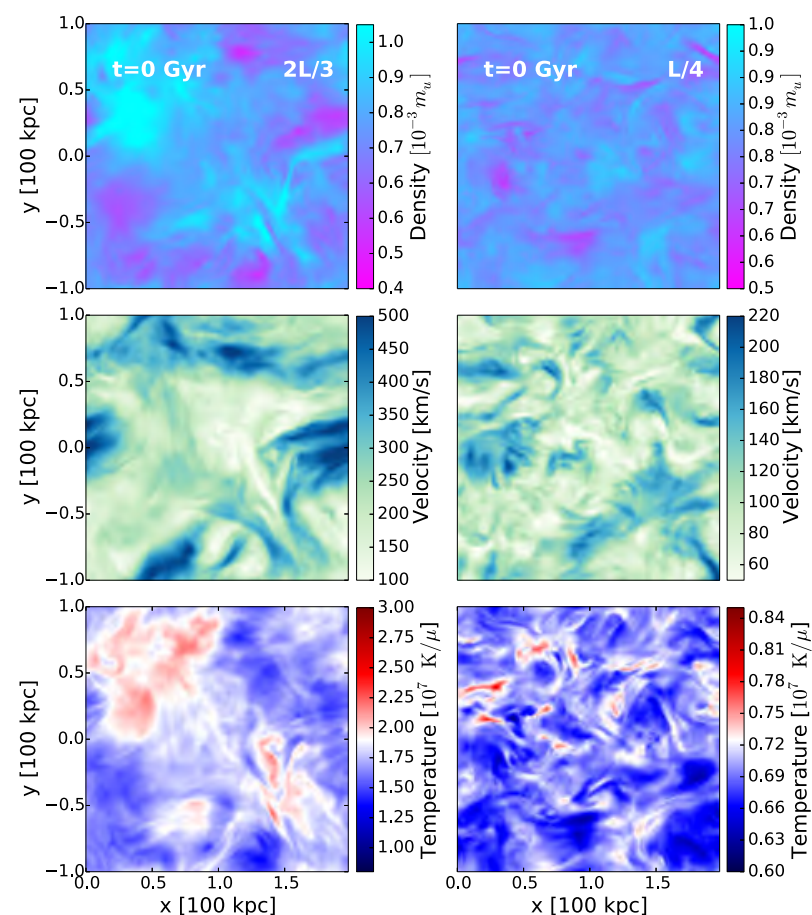

Figure 5. Projected maps along the $z$-axis of the different initial conditions (25 kpc slice), where $m_{u}$ is the atomic mass unit and $\mu$ is the mean molecular weight. Here, we show the right-hand half-size of the box containing the turbulent media. From top to bottom the reader can see the average density, velocity, and temperature field for the $2 L / 3$ (left-hand column) and $L / 4$ (righthand column) cases, where $2 L / 3$ and $L / 4$ refer to the integral scale of each type of turbulence $(L=200 \mathrm{kpc})$.

re-write equation (11) making use of the Rankine-Hugoniot jump equations (see derivation in Blandford \& Eichler 1987):

$q=\frac{3\left(\gamma_{0}+1\right) \mathcal{M}^{2}}{2\left(\mathcal{M}^{2}-1\right)}=\frac{4 \mathcal{M}^{2}}{\mathcal{M}^{2}-1}$,

where for the second equality we have considered an adiabatic index of $\gamma_{0}=5 / 3$. The corresponding power-law index for the macroparticle distribution function can be also obtained by assuming isotropy, i.e. $N(p, \tau)=\int \Omega_{\tau} p^{2} f \approx 4 \pi p^{2} f$, where $\mathrm{d} \Omega_{\tau}$ is the solid angle around the direction $\tau$. Since the particles are relativistic, we have that $N(E, \tau) \mathrm{d} E=N(p, \tau) \mathrm{d} p$. Moreover, from the isotropic condition we also have that $N(E)=4 \pi N(E, \tau)$.
Therefore, the tracer particle energy distribution function at the activation time is given by

$\chi(E)=\frac{N(E)}{n_{0}}=\frac{N_{0}}{n_{0}} E^{-p}$,

where $p=q-2$ is what it is usually called the injection spectral index, $N_{0}$ is the normalization factor and $n_{0}$ is the fluid number density. $N_{0}$ is assigned according to the energy contained in the shock. That is, we considered that the total energy per fluid number density is

$\int \chi(E) E \mathrm{~d} E=\frac{E_{\mathrm{tot}}}{n_{0}}$,

where $E_{\text {tot }}=\eta E_{\text {shock }}=\eta \rho_{\text {post }} v_{\text {shock }}^{2}$ and $\eta$ is the acceleration efficiency. For which finally one can obtain the normalization factor:

$N_{0}=\left\{\begin{array}{ll}\frac{\eta E_{\text {shock }}(4-q)}{\left[E_{\max }{ }^{4-q}-E_{\min }{ }^{4-q}\right]} & \text { if } q \neq 4 \\ \eta E_{\text {shock }} \log \left(\frac{E_{\max }}{E_{\min }}\right) & \text { if } q=4\end{array}\right.$.

The PLUTO code allows us to compute the maximum and minimum energy at each time-step. The maximum energy $E_{\max }$ is imposed considering the maximum allowed Larmor radius for each particle. The minimum energy $E_{\min }$ is estimated balancing the synchrotron and acceleration time-scales (see full explanation in Böttcher \& Dermer 2010; Mimica \& Aloy 2012; Vaidya et al. 2018) so it depends on the acceleration efficiency. Moreover, the acceleration efficiency in collisionless relativistic shocks can also depend on the energy of the particles (see Sironi, Spitkovsky \& Arons 2013). In this work, for simplicity we assume a fixed acceleration efficiency of $\eta=10^{-3}$ and fixed energy limits of $\gamma_{\min }=1$ and $\gamma_{\max }=10^{5}$. This acceleration efficiency agrees with the expectations of DSA for strong shocks (e.g. Kang, Ryu \& Jones 2012) and lies in the range of values required to explain observations of radio relics (see Botteon et al. 2020). Nevertheless, this means that the final synchrotron emission obtained can be re-scaled to the desired $\eta$ since in our case the energy limits remain constant.

\subsection{Synchrotron emission}

The synchrotron emission of a tracer particle in a local magnetic field $\boldsymbol{B}^{\prime}$ in the direction $\hat{\boldsymbol{n}}_{\text {los }}{ }^{\prime}$, per unit solid angle, volume and frequency is given by

$\mathcal{J}_{\text {syn }}^{\prime}\left(v_{\text {obs }}^{\prime}, \hat{\boldsymbol{n}}_{\text {los }}^{\prime}, \boldsymbol{B}^{\prime}\right)=\int N\left(E^{\prime}\right) \mathcal{P}\left(v_{\mathrm{obs}}^{\prime}, E^{\prime}, \phi^{\prime}\right) \mathrm{d} E^{\prime} \mathrm{d} \Omega^{\prime}$, 
where $\mathcal{P}\left(v_{\mathrm{obs}}^{\prime}, E^{\prime}, \phi^{\prime}\right)$ is the synchrotron power per unit frequency and unit solid angle emitted by a single particle that has energy $E^{\prime}$ and $\phi^{\prime}$ is the angle that the velocity vector of the particle makes with the direction $\hat{\boldsymbol{n}}_{\text {los }}^{\prime}$. Following Ginzburg \& Syrovatskii (1965), we get

$\mathcal{J}_{\text {syn }}^{\prime}\left(v_{\mathrm{obs}}^{\prime}, \hat{\boldsymbol{n}}_{\text {los }}^{\prime}, \boldsymbol{B}^{\prime}\right)=\frac{\sqrt{3} e^{3}}{4 \pi m_{e} c^{2}}\left|\boldsymbol{B}^{\prime} \times \hat{\boldsymbol{n}}_{\text {los }}{ }^{\prime}\right| \int N\left(E^{\prime}\right) F(\xi) \mathrm{d} E^{\prime}$,

where $\hat{\boldsymbol{n}}_{\boldsymbol{l o s}}{ }^{\prime}$ is the unit vector in the direction of the line of sight in the comoving frame and $F(\xi)$ is a Bessel function integral given by

$F(\xi)=\xi \int_{\xi}^{\infty} K_{5 / 3}\left(z^{\prime}\right) \mathrm{d} z^{\prime}$,

where

$\xi=\frac{v_{\mathrm{obs}}^{\prime}}{v_{c}^{\prime}}=\frac{4 \pi m_{e}^{3} c^{5} v_{\mathrm{obs}}^{\prime}}{3 e E^{\prime 2}\left|\boldsymbol{B}^{\prime} \times \hat{\boldsymbol{n}}_{\mathrm{los}}^{\prime}\right|}$,

where $v_{c}^{\prime}$ is defined as the critical frequency at which the emission peaks. Note that only those particles with a pitch angle coinciding with the angle between $\boldsymbol{B}^{\prime}$ and $\hat{\boldsymbol{n}}_{\text {los }}^{\prime}$ contribute to the emission along the line of sight in equation (17).

The emissivity in equation (17) is measured in the local comoving frame with the emitting volume. If we want the emissivity in a fixed observer's frame then we have to apply a transformation: ${ }^{4}$

$\mathcal{J}_{\text {syn }}\left(v_{\text {obs }}, \hat{\boldsymbol{n}}_{\text {los }}, \boldsymbol{B}\right)=\mathcal{D}^{2} \mathcal{J}_{\mathrm{syn}}^{\prime}\left(v_{\mathrm{obs}}^{\prime}, \hat{\boldsymbol{n}}_{\text {los }}{ }^{\prime}, \boldsymbol{B}^{\prime}\right)$,

where $\mathcal{D}$ is a Doppler factor given by

$\mathcal{D}=\frac{1}{\gamma\left(1-\hat{\boldsymbol{n}}_{\text {los }} \cdot \boldsymbol{v}\right)}$,

where $\gamma$ is the Lorentz factor of the tracer particle. The unit vectors on the direction of the line of sight in the comoving and observer's frame are related through

$\hat{\boldsymbol{n}}_{\mathrm{los}}^{\prime}=\mathcal{D}\left[\hat{\boldsymbol{n}}_{\mathrm{los}}+\left(\frac{\gamma^{2}}{\gamma+1} \boldsymbol{v} \cdot \hat{\boldsymbol{n}}_{\mathrm{los}}-\gamma\right) \boldsymbol{v}\right]$.

\subsection{Radio emission maps}

In the preceding equations, the vector $\hat{\boldsymbol{n}}_{\text {los }}$ can be selected according to an observing angle $\theta_{\mathrm{obs}}$ with respect to the line of sight. In this work, we only show results considering $\theta_{\text {obs }}=0^{\circ}$, that is we consider an observer's reference frame in which $z$ lies along the line of sight $\hat{\boldsymbol{n}}_{\mathrm{los}}$ and $x$ and $y$ are in the plane of the sky. The specific intensity (or surface brightness) maps can then be obtained by integrating along a line of sight as

$I_{v}=\int \mathcal{J}_{\text {syn }}\left(v_{\text {obs }}, x, y, z\right) \mathrm{d} z$

in units of erg $\mathrm{cm}^{-2} \mathrm{~s}^{-1} \mathrm{~Hz}^{-1} \mathrm{str}^{-1}$. This is doable due to the fact that the emissivity information $\mathcal{J}_{\text {syn }}$ of the Lagrangian tracer particles is interpolated back on to the Eulerian grid. It is also possible to obtain spectral index maps by means of

$-\alpha(x, y)=\frac{\log \left[I_{v_{2}}(x, y) / I_{\nu_{1}}(x, y)\right]}{\log \left(v_{2} / v_{1}\right)}$,

and the integrated spectra (or net flux) can be obtained by integrating the specific intensity $I_{v}$ over the area covered by the source in the

${ }^{4}$ The reader should note that the primed quantities in equation (20) refer to the comoving frame, whereas standard notation refers to the observer's frame. plane of the sky, that is

$F_{v}(v)=\int I_{v}(v, x, y) \mathrm{d} x \mathrm{~d} y$,

in units of $\mathrm{erg} \mathrm{s}^{-1} \mathrm{~Hz}^{-1} \mathrm{str}^{-1}$.

\section{RESULTS}

\subsection{Fluid properties}

In this subsection, we describe some of the features of the fluid starting with the evolution of velocity, temperature, and magnetic field, for the $2 L / 3, \mathcal{M}=3$, and $\theta_{\mathrm{bn}}=90^{\circ}$ case (see Fig. 6). In all of our runs, the initial shock sweeps region II maintaining a constant velocity and planar shape due to the fact that this region is initially uniform. Next, the shock enters region III, where the shock will no longer be uniform and be affected by the anisotropies of the fluid. The two first rows in Fig. 6 highlight how weak internal shocks and turbulence are generated in the downstream as the main shock travels through the simulation box. The third row shows the streamlines of the magnetic field. The streamlines are coloured according to the magnitude of the $y$-component of the magnetic field, $B_{y}$, to illustrate how the shock compression amplifies the field. The fourth row shows 1D profiles of the magnetic field obtained by integrating along the LOS. As expected from the MHD Rankine-Hugoniot conditions, the component parallel to the shock normal, $B_{x}$, is conserved, while the other components are amplified and stretched as a result of shock compression. We show also the magnetic field profile weighted with the synchrotron emission at $150 \mathrm{MHz}$ (blue solid lines).

In Fig. 7 (see Table 2 for the values used in Figs 7 and 8), we show how the standard deviation (volumetric value) of the magnetic and velocity field evolve for all runs listed in Table 1 . The evolution is characterized by two phases: (1) a first phase in which the shock is crossing region II (lasting 50-150 Myr depending on the type of run), and we have a purely decaying turbulence system on the right-hand side of the box $([0,200] \mathrm{kpc}$, see Fig. 4), and (2) a second phase in which the shock has already entered region III and is compressing the turbulent medium. The dashed grey vertical lines in Fig. 7 define the beginning of this second phase (the time differs according to the initial Mach number of each run).

The evolution of the standard deviation of the velocity and magnetic field are in general not correlated (see third column of Fig. 7). We also analysed the standard deviation of the parallel and perpendicular components of both fields with respect to the shock normal. In general, we find that the evolution of the parallel component dominates the evolution of $\sigma_{V}$, whereas the perpendicular component dominates the evolution of $\sigma_{B}$. The standard deviation of the parallel component (see first column of Fig. 7) decreases with time for both fields. The standard deviations of the perpendicular components (see second column of Fig. 7) show a different evolution: the perpendicular component, $\sigma_{V_{\perp}}$, follows the same behaviour of $\sigma_{V}$, while the perpendicular $\sigma_{B}$ always increases right after the shock crossing in all runs. This trend is persistent and gets stronger with higher resolution (see Fig. C1 in Appendix C).

The reason for this is that most velocity fluctuations are driven in the direction parallel to the shock normal, while magnetic field fluctuations are initially driven perpendicular to the shock normal due to compression. With time, the dynamics gets more complicated due to the shock compression and possibly also stretching of the magnetic field. The increase in $\sigma_{B}$ induces a delay in the velocity field dissipation. The plateau observed in $\sigma_{V}$ in the second phase (evolution to the right of the dashed grey lines in Fig. 7) indicates 


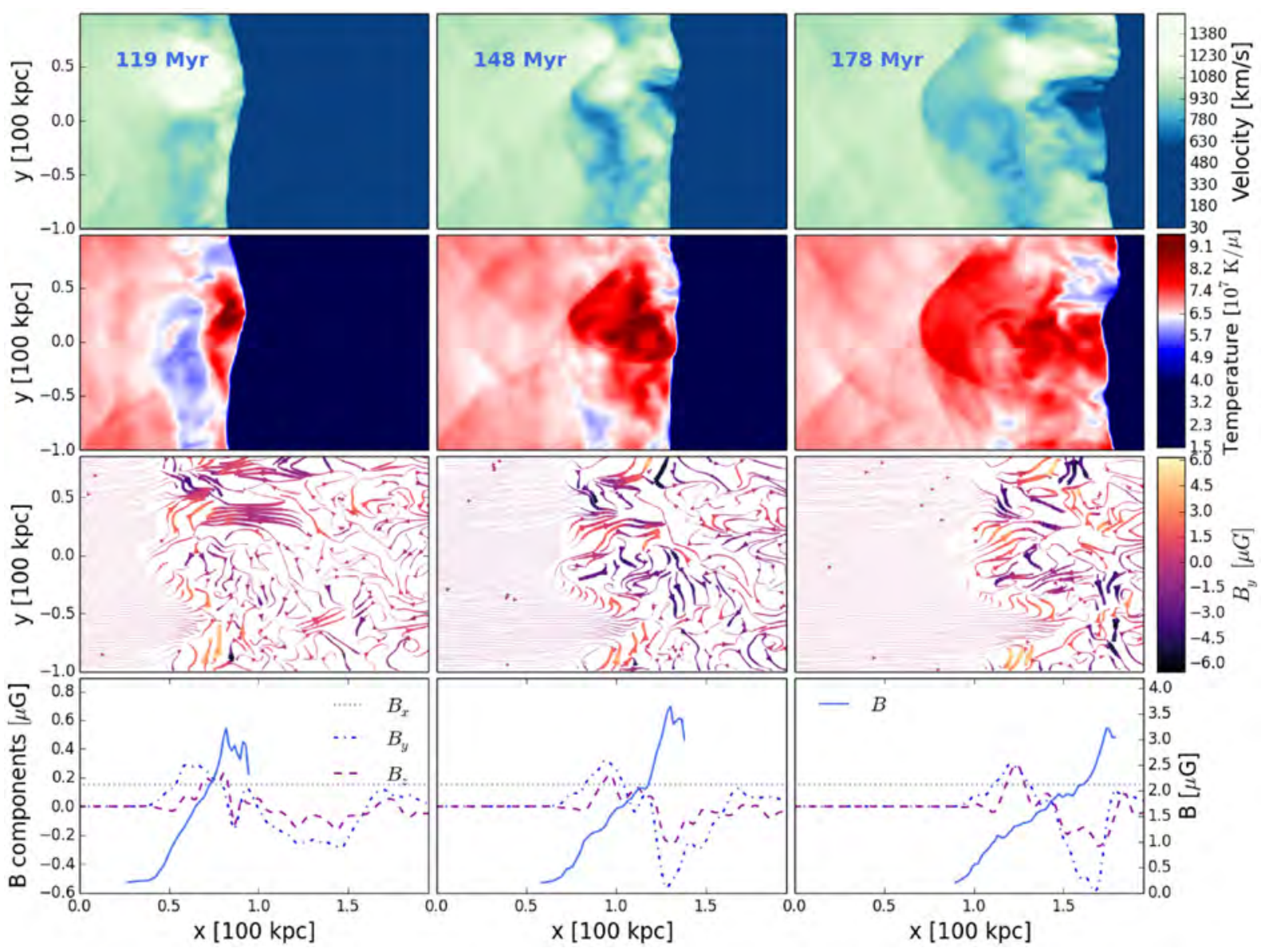

Figure 6. Evolution of the $2 L / 3, \mathcal{M}=3$, and $\theta_{\mathrm{bn}}=90^{\circ}$ case. First row: $20 \mathrm{kpc}$ slice along the $z$-axis of velocity field. Second row: $20 \mathrm{kpc}$ slice along the $z$-axis of temperature Third row: streamlines of total magnetic field (note that the colour-code only denotes the strength of $y$-component). Fourth row: 1D magnetic profiles corresponding. The component's profiles have no weighting, whereas the magnetic field strength, $B$, is weighted with the $150 \mathrm{MHz}$ synchrotron emissivity.

that the shock-induced turbulence could be maintained only for some time before $\sigma_{V}$ decreases due to turbulent dissipation.

For all our runs, we verified that the density and also the temperature evolve in the same fashion as the velocity field in Fig. 7 and it is only the standard deviation of the magnetic field that has a characteristic evolution in both phases.

In the following, we summarize the observed effects for this second phase:

(i) The role of different turbulent injection scales: We find that $\sigma_{B}$ decreases faster in a system with smaller magnetic fluctuations $(L / 4)$ than in one with larger fluctuations $(2 L / 3)$. This is expected as the turbulence injection scale is smaller in the $L / 4$ case and therefore the eddy-turnover time is shorter. In fact, in the $L / 4$ case, the shockinduced turbulence seems to only have an increasing effect on $\sigma_{B}$ whenever the shock is perpendicular, i.e. $\theta_{\mathrm{bn}}=90^{\circ}$.

(ii) The role of the main shock's Mach number: The largest impact on increasing $\sigma_{B}$ is due to a larger Mach number. For example, the $\mathcal{M}=3$ and $\theta_{\mathrm{bn}}=0^{\circ}$ case shows an increase of $\sim 31$ per cent for the $2 L / 3$ turbulence and $\sim 7$ per cent for the $L / 4$ turbulence. Conversely, the $\mathcal{M}=2$ and $\theta_{\mathrm{bn}}=0^{\circ}$ case shows an increase of $\sim 25$ per cent for the $2 L / 3$ turbulence and a decrease of $\sim 4$ per cent for the $L / 4$ turbulence. This suggests that weak shocks $(\mathcal{M}=2)$ are less likely to modify the initial distribution of magnetic fields for smaller turbulent scales. (iii) The role of obliquity: A perpendicular shock has the strongest effect on broadening the downstream magnetic field distribution. This is expected as the perpendicular components of the magnetic field are the only ones affected by the shock compression. The largest increase in both runs is $\sim 38$ per cent (with respect to the corresponding dashed grey vertical line in Fig. 7).

Finally, we show in Fig. 8 the profiles of the total magnetic field strength for all runs for two snapshots. These 1D profiles are obtained integrating along the LOS and then taking an average. Each profile is again normalized to the pre-shock magnetic field of each run, $B_{\text {pre }}$. The two times shown in Fig. 8 correspond to when the shock is starting to compress the turbulent medium (left-hand panel of Fig. 8), and when the shock has crossed the whole simulation box (right-hand panel of Fig. 8). The overall magnetic amplification in the downstream region differs in each run. The discrepancies observed in Fig. 8 can be explained by the different distribution of incidence angles between the upstream magnetic field and the shock normal along the shock front in the different runs. The downstream region in the $\mathcal{M}=3$ case develops very similar profiles for both types of turbulence. In particular, the $L / 4$ case leads to higher magnetic amplification (compare blue and red lines), whereas the $2 L / 3$ case leads to comparable downstream magnetic profiles (compare purple and orange lines). We observe that a lower Mach number, such as $\mathcal{M}=2$, leads to less magnetic amplification, owing to the lower shock compression factor. In this case, the final extent of the region 

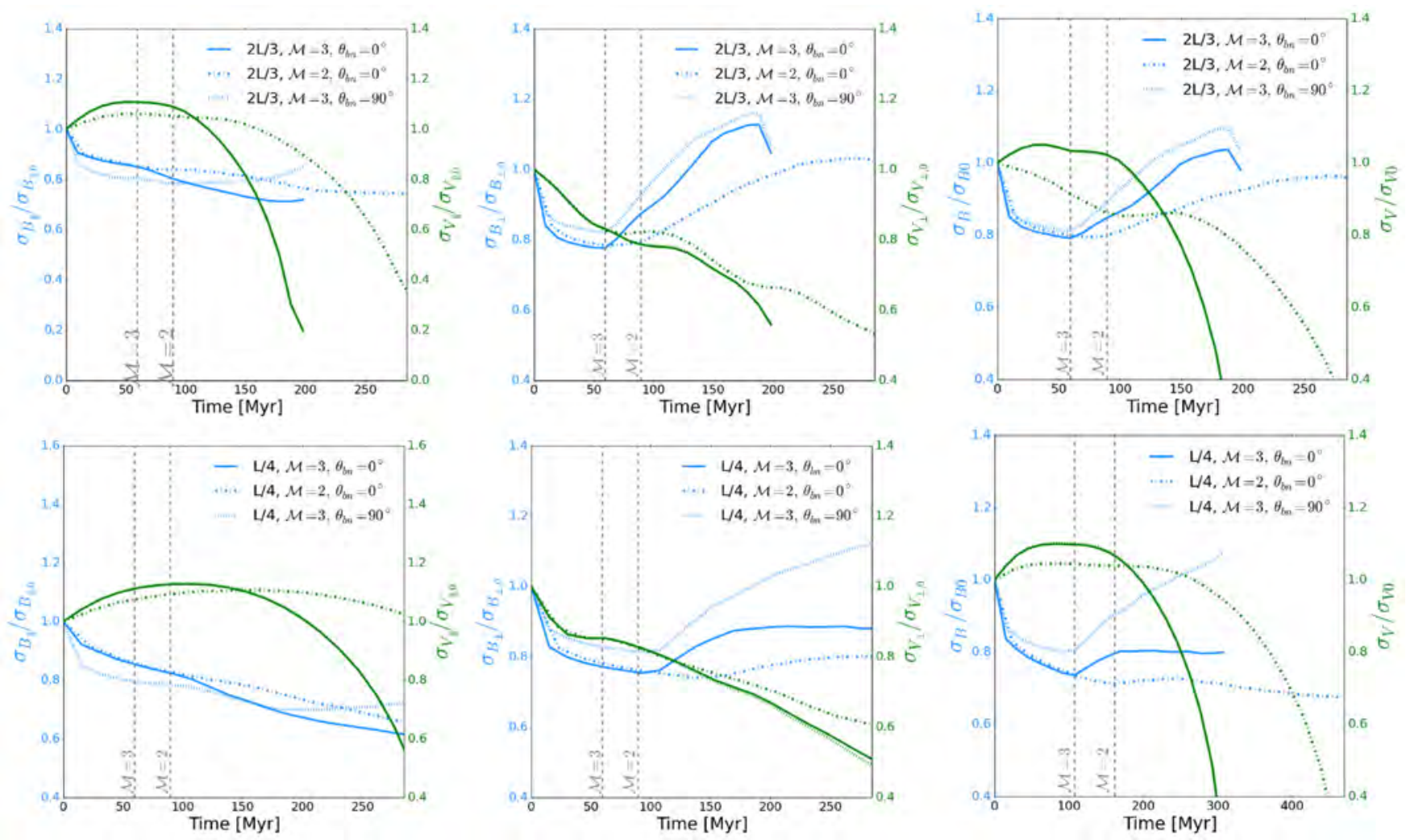

Figure 7. Evolution of the standard deviation of the magnetic field (left axis) and velocity field (right axis). Top panels: $2 L / 3$ case. Bottom panels: $L / 4$ case. First column: parallel component to the shock, i.e. $B_{\|}=B_{x}$ and $v_{\|}=v_{x}$. Second column: perpendicular component, i.e. $B_{\perp}=\sqrt{B_{y}^{2}+B_{z}^{2}}$ and $v_{\perp}=\sqrt{v_{y}^{2}+v_{z}^{2}}$. Third column: standard deviation of the magnetic and velocity field strengths. $\sigma_{B}$ and $\sigma_{V}$ are normalized to their value at $t=0$ for purposes of comparison between different runs (see Table 2).

Table 2. Values used in Figs 7 and 8. The second and third columns are the initial standard deviation of the velocity and magnetic field in the whole simulation box. The fourth column shows the total time needed for the shock to cross the entire simulation box.

\begin{tabular}{lccc}
\hline Run ID & $\sigma_{V 0}\left(\mathrm{~km} \mathrm{~s}^{-1}\right)$ & $\sigma_{B 0}(\mu \mathrm{G})$ & $t_{\text {shock }}(\mathrm{Myr})$ \\
\hline k1p5_M2_parallel & 217.7 & 1.016 & 282 \\
k1p5_M3_parallel & 388.5 & 1.016 & 188 \\
k4_M2_parallel & 133.1 & 0.659 & 439 \\
k4_M3_parallel & 246.1 & 0.513 & 292 \\
k1p5_M3_perpendicular & 388.5 & 1.099 & 188 \\
k4_M3_perpendicular & 246.1 & 0.513 & 293 \\
\hline
\end{tabular}

where a magnetic field amplification occurs in the downstream is $\sim 25 \mathrm{kpc}$ larger than in the case with a $\mathcal{M}=3$ shock. This means that the strength of this shock is insufficient to further compress the turbulent medium, thus producing a more extended turbulent magnetic region which will be also reflected in the synchrotron emission.

\subsection{Emission}

We present a few three-dimensional renderings of the synchrotron emission produced by our modelling in Fig. 9, as seen along different lines of sight. Although the radio emission seems fairly uniform when observed edge-on (see following Fig. 10), the emission is not spatially uniform, but concentrated into threads and filaments in the shock plane. The combination of shock compression and turbulence introduces anisotropies and fluctuations in the flow. This, in turn,

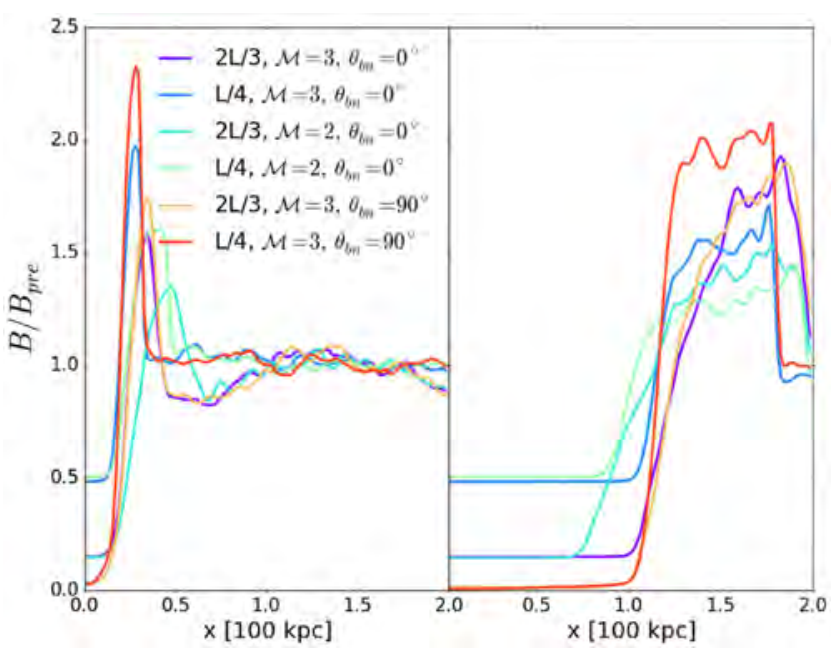

Figure 8. 1D magnetic field profile of all runs. Left-hand panel: Shock front just entering the turbulent medium at $t_{\text {shock }} / 9$. Right-hand panel: Shock front is almost at the right end of the $x$-axis at $t_{\text {shock }} / 19$. Refer to Table 2 for the value of $t_{\text {shock }}$ of each run.

directly affects the advection properties and energy evolution of the CR particles.

We show the surface brightness maps at $150 \mathrm{MHz}$ for all runs at a time when the shock front has reached almost the right end of the simulation box in Fig. 10 (the different times are specified in 

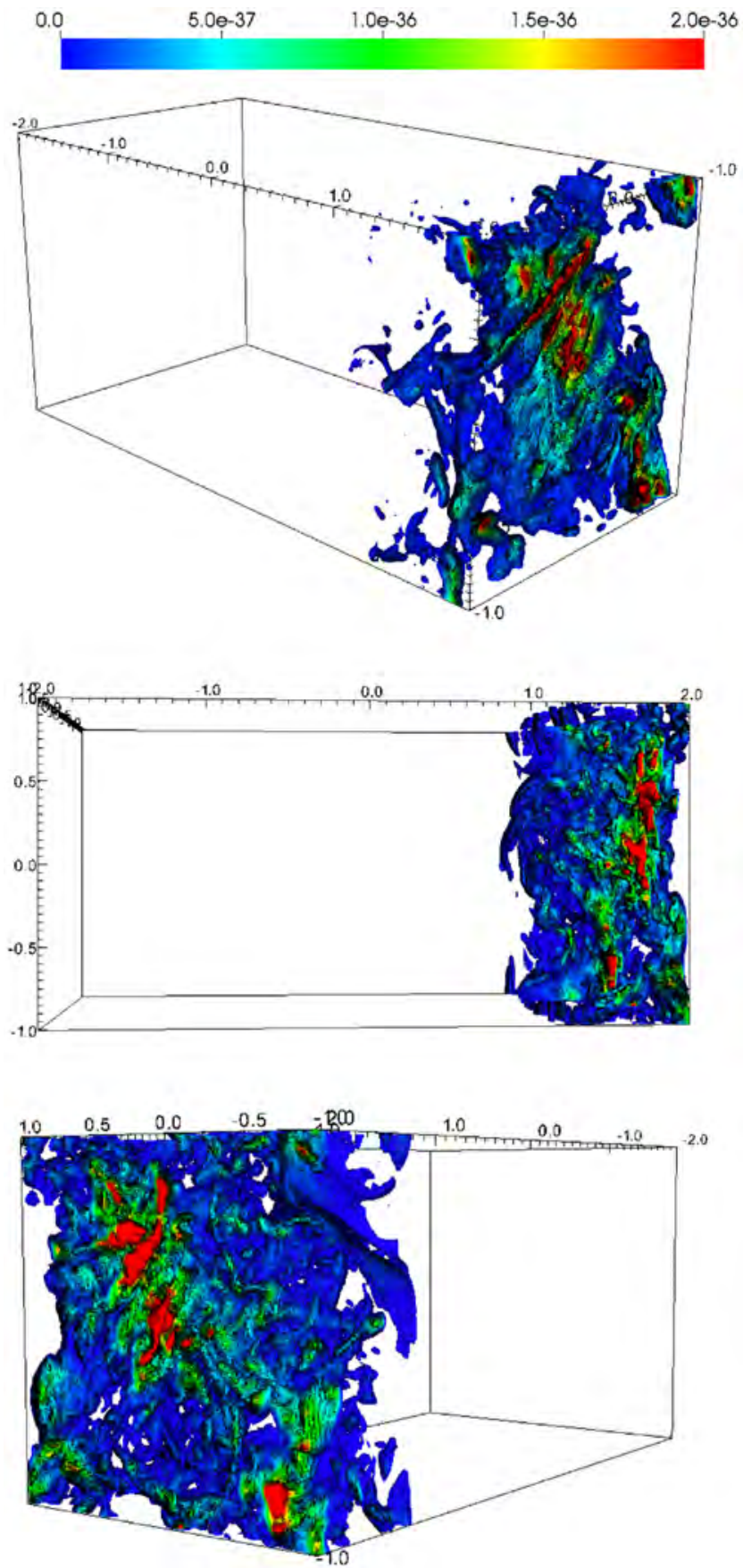

Figure 9. Visualization of synchrotron emissivity $\mathcal{J}_{\text {syn }}$ isocurves for the $2 L / 3, \mathcal{M}=3$, and $\theta_{\mathrm{bn}}=0^{\circ}$ run at $t=178 \mathrm{Myr}$. The emissivity is shown in units of erg $\mathrm{cm}^{-3} \mathrm{~s}^{-1} \mathrm{~Hz}^{-1} \mathrm{str}^{-1}$. The axis are shown in units of $100 \mathrm{kpc}$. See online supplementary material for the online video.

the upper left corner of each panel). ${ }^{5}$ Note that we only applied Gaussian smoothing for the surface brightness maps in Fig. 10 (meant to mimic the finite spatial resolution of a typical LOFARHBA observation), while all of our following analysis was done without any smoothing. In the 3D view presented in Fig. 9, we can distinguish the complex substructure of the emission in the form of filaments, bristles, ribbons or other structures that cannot be classified

${ }^{5}$ We have included the surface brightness maps along the $x$-axis in Appendix D for completeness. in a single group. Nevertheless, since the emissivity is not volumefilling and the strength of the emission varies from region to region, some of this structure vanishes in projection.

The morphology observed in Figs 9 and 10 depends on three factors: the strength of the shock, the obliquity of the shock, and the type of turbulence. A higher Mach number leads to stronger emission and elongated patterns due to a stronger compression of the magnetic field. On the other hand, the role of the upstream turbulence is more complex.

One can observe the effect of more elongated patterns for the cases with a shock of $\mathcal{M}=3$ [comparing panels (a), (c), (d), and (f) of Fig. 10] due to the increased stretching of the eddies.

On the other hand, a shock of strength $\mathcal{M}=2$ can produce disrupted patterns in our simulated emission. The shock front is less likely to look totally disrupted in the $L / 4$ case [see panel (e)], while this effect is particularly noticeable in the $2 L / 3$ case [see panel (b)]. We notice that this is a direct consequence of the sound speed in the media. For example, the $2 L / 3$ turbulence easily leads to regions with Mach numbers lower than our threshold $\left(\mathcal{M}_{\min }=1.3\right.$, see Appendix B). This happens then because the initial sound speed of the $2 L / 3$ turbulence is slightly higher than that of the $L / 4$ case (see Section 2.1). Subsequently, turbulent dissipation leads to an increase in sound speed and therefore lowering the Mach number. Such low Mach numbers are not expected to accelerate electrons via the DSA process and therefore they are excluded from our modelling. In fact, Kang, Ryu \& Ha (2019) found that quasi-perpendicular shocks with $\mathcal{M} \lesssim 2.3$ may not efficiently accelerate electrons through DSA. Assessing the impact of different turbulent injection scales in $\mathcal{M}=2$ shocks, would then require a tailored set-up which we leave for future work.

Finally, obliquity produces more elongated emission because shock compression only amplifies the component parallel to the shock front (i.e. the $B_{y}$ and $B_{z}$ components in this study).

In Fig. 11, we show 1D profiles of the emission presented in Fig. 10, at two more observing frequencies: $1.5 \mathrm{GHz}$ and $650 \mathrm{MHz}$. The most extended emission is found in the runs with $\mathcal{M}=2$ (see bottom panel of Fig. 11) in agreement with the 1D magnetic profiles previously shown in Fig. 8. These low Mach number runs also show the lowest values of surface brightness along the downstream due to the initial normalization depending on the Mach number. This suggests that, in our survey of merger shocks, only those with $\mathcal{M} \sim 2$ would require a higher acceleration efficiency to reach observable values as it has been pointed out in previous works (e.g. Botteon et al. 2020). Finally, the steepness of the emission profile depends on the magnetic morphology. Comparing all the $\mathcal{M}=3$ runs, the $2 L / 3$ turbulence case shows a shallower decline compared to the $L / 4$ case. This is a direct consequence of the initial distribution of the magnetic field strength. In Fig. 3 of Section 2.1, we showed that initially the $L / 4$ run reaches higher magnetic field values in the tail of its PDF, which leads to larger synchrotron losses.

\subsubsection{Spectral index}

In Fig. 12, we show the spectral index maps for the two different turbulent media with the $\mathcal{M}=3$ shock using equation (24). We can observe the expected spectral index gradient starting from the shock front (red) to the end of the downstream region (blue). In the $2 L / 3$ case, the spectral index values range between $\alpha=-0.6$ and $\alpha=$ -4.9 , while it goes from $\alpha=-0.6$ to $\alpha=-6.9$ in the $L / 4$ case. This agrees with the previous subsection, where we discussed the emission profiles. In the same way, the turbulent medium with smaller initial fluctuations $L / 4$ is more likely to produce a steeper gradient because the initial magnetic field distribution has a larger tail (see Fig. 3). 
(a) $2 \mathrm{~L} / 3, \mathcal{M}=3, \theta_{b n}=0^{\circ}$

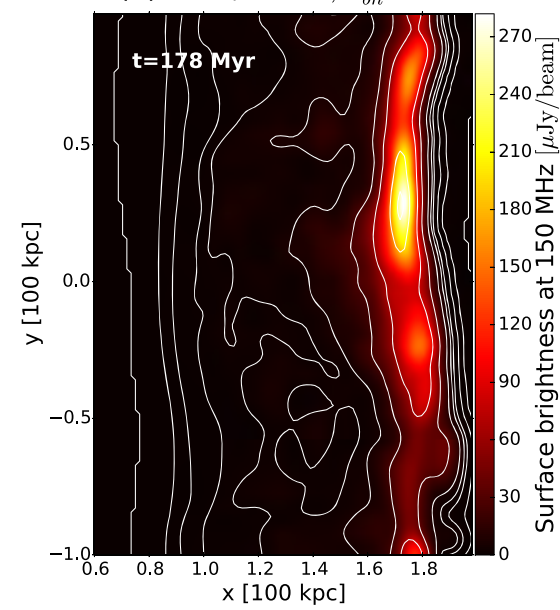

(d) $\mathrm{L} / 4, \mathcal{M}=3, \theta_{b n}=0^{\circ}$

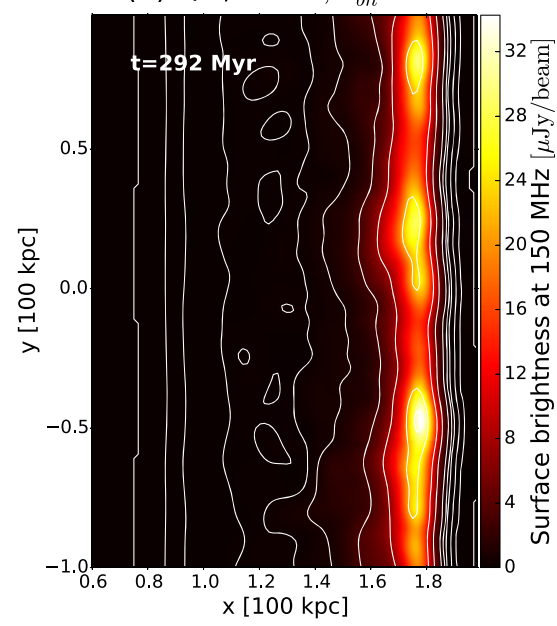

(b) $2 \mathrm{~L} / 3, \mathcal{M}=2, \theta_{b n}=0^{\circ}$

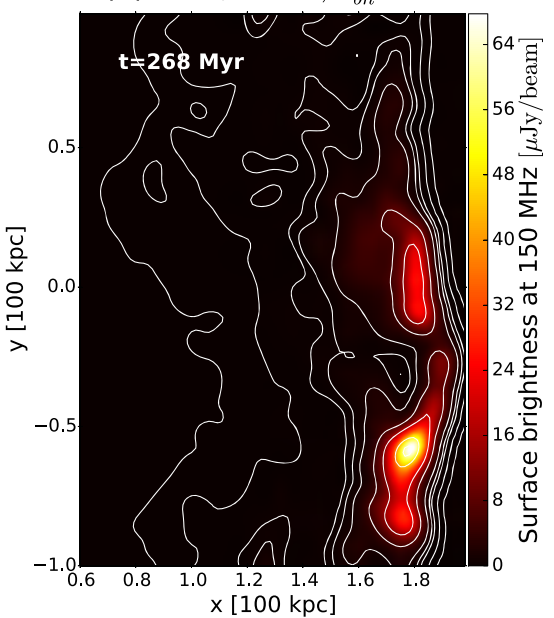

(e) $\mathrm{L} / 4, \mathcal{M}=2, \theta_{b n}=0^{\circ}$

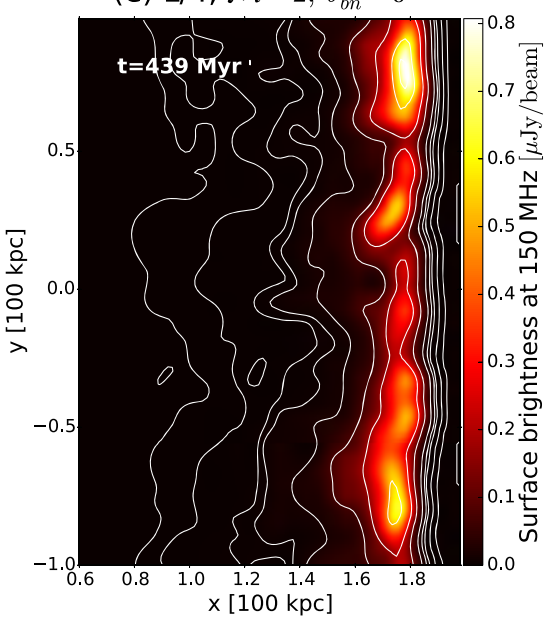

(c) $2 \mathrm{~L} / 3, \mathcal{M}=3, \theta_{b n}=90^{\circ}$

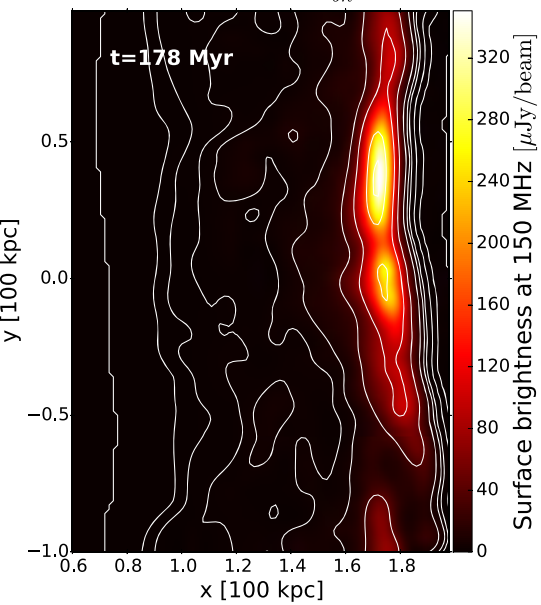

(f) $\mathrm{L} / 4, \mathcal{M}=3, \theta_{b n}=90^{\circ}$

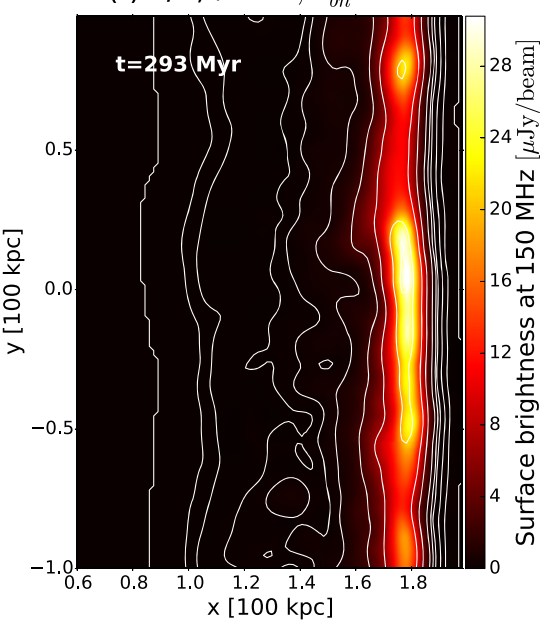

Figure 10. Surface brightness at $150 \mathrm{MHz}$ for all runs in Table 1 (see equation 23). We considered a beam of $\theta^{2}=15$ arcsec $\times 15$ arcsec to get the surface brightness $\left(\theta^{2} I_{v}\right)$ in units of $\mu \mathrm{Jy}_{\text {beam }}{ }^{-1}$. We smoothed the maps with a Gaussian kernel with FWHM $=7.24 \mathrm{kpc}(\operatorname{assuming} z=0.023)$.

In Fig. 13, we show the corresponding spectral index profiles along the downstream region for completeness. In the top panel of Fig. 13 we show how the profile changes when taking into account different frequencies for one specific run: $2 L / 3, \mathcal{M}=3$, and $\theta_{\mathrm{bn}}=$ $0^{\circ}$. The profiles start to differ beyond $\sim 20 \mathrm{kpc}$ from the shock front where the emission at lower frequencies decreases more slowly. In the lower panel, we show the spectral index profiles for all of our runs between 650 and $150 \mathrm{MHz}$. The selected snapshots correspond to those in Fig. 10. For the higher Mach number (i.e. $\mathcal{M}=3$ ), the $2 L / 3$ profiles agree more with observations than the $L / 4$ profiles. In the $L / 4$ case, the spectral index profiles are steeper than observed. For example, the relic in the cluster MACS J0717.5+3745 (see van Weeren et al. 2017; Bonafede et al. 2018) with a Mach number of $\mathcal{M}=2.7$ (inferred from the injection spectral index $\alpha$ ), shows a spectral index steepening up to values of $\sim-2.5$ over a region of $\lesssim 170 \mathrm{kpc}$. Another example is the 'Toothbrush' relic (see Rajpurohit et al. 2018, 2020) which steepens also up to values of $\sim-2.5$ over a region of $\sim 500 \mathrm{kpc}$. This suggests that the initial turbulent magnetic field distribution before a shock crossing is rather narrow. For the forced turbulence used in this work, this means that the standard deviation must be smaller than $\sigma_{B} \sim 1 \mu \mathrm{G}$ (see Fig. 3 in Section 2.1 for the initial magnetic field distribution). On the other hand, this also suggests that the injection scale (and also the magnetic coherence scale) of the turbulence in galaxy clusters outskirts could be $2 L / 3$ $(\sim 133 \mathrm{kpc})$ or even larger.

It is fairly common in observations of radio relics, that only the integrated spectral index can be computed. This is done by fitting the total observed flux (see equation 25) against different available frequencies. We computed the integrated spectral index in this fashion using $1.5 \mathrm{GHz}, 650 \mathrm{MHz}$, and $150 \mathrm{MHz}$ frequencies. In Fig. 14, we show how the integrated spectral index evolves as the shock sweeps across the simulation box. In the first $\sim 60 \mathrm{Myr}$ the integrated spectral indices differ quite significantly, whereas after $\sim 140$ Myr the value of the integrated spectra seems to converge to the same value for all runs. The relation between the real spectral index $\alpha$ and the integrated one is often assumed to be (e.g. Kardashev 1962; Heavens \& Meisenheimer 1987)

$\alpha_{\text {int }}=\alpha+\frac{1}{2}=\frac{\mathcal{M}^{2}+1}{\mathcal{M}^{2}-1}$,

and therefore we also plot the expected $\alpha_{\text {int }}$ for different Mach numbers as a reference in Fig. 14 with grey dashed horizontal lines. The integrated spectral index from our runs does not follow a characteristic pattern and it does not remain strictly constant through a time span of roughly 200-300 Myr. In addition, we include the corresponding evolution of the integrated spectral index for two extra 


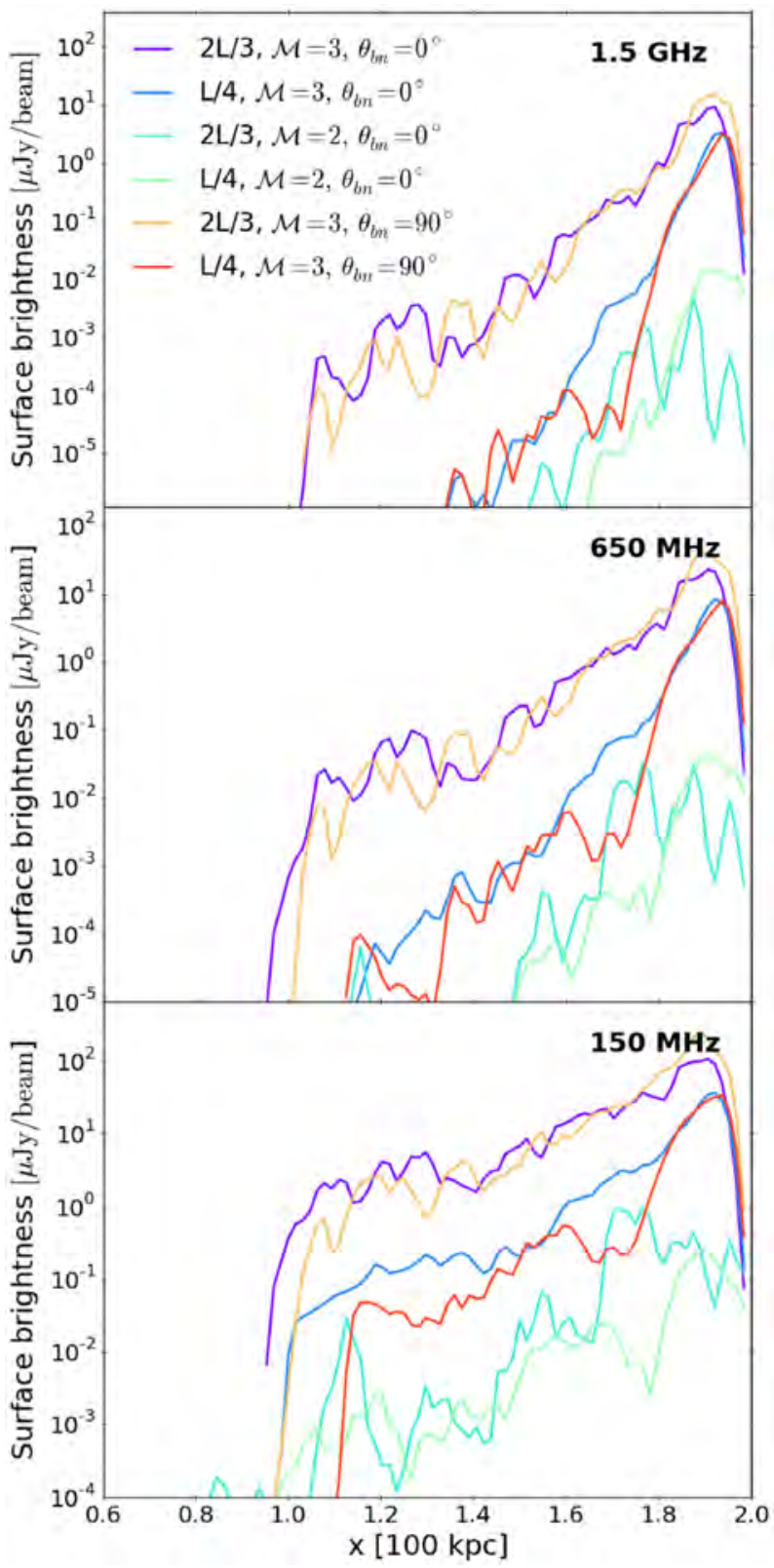

Figure 11. 1D surface brightness profiles obtained from the emission maps at $1.5 \mathrm{GHz}$ (top panel), $650 \mathrm{MHz}$ (middle panel), and $150 \mathrm{MHz}$ (bottom panel) for all runs.

runs with a completely uniform medium (density, velocity, pressure and magnetic field) with shocks of strength $\mathcal{M}=2$ and $\mathcal{M}=3$. In the uniform media, equation (26) holds after $\sim 50 \mathrm{Myr}$ when the energy spectrum reaches a steady state at the shock (see Kang, Ryu \& Jones 2017 for a one-dimensional uniform media study), however this is not the case for all the turbulent media. In particular, the effect of turbulence on the 3D distribution of the synchrotron emissivity is that it makes it patchy and not volume-filling (as can be observed in Fig. 9). This in turn has an effect on the integrated flux and therefore the integrated spectral index.

Hence, it can be difficult to recover the real spectral index and Mach number through this method, even if recent high-resolution observations of radio relics show consistent integrated and injection spectra (e.g. Rajpurohit et al. 2020). The fact that equation (26) only holds for a planar shock where all the fields are uniform, suggests
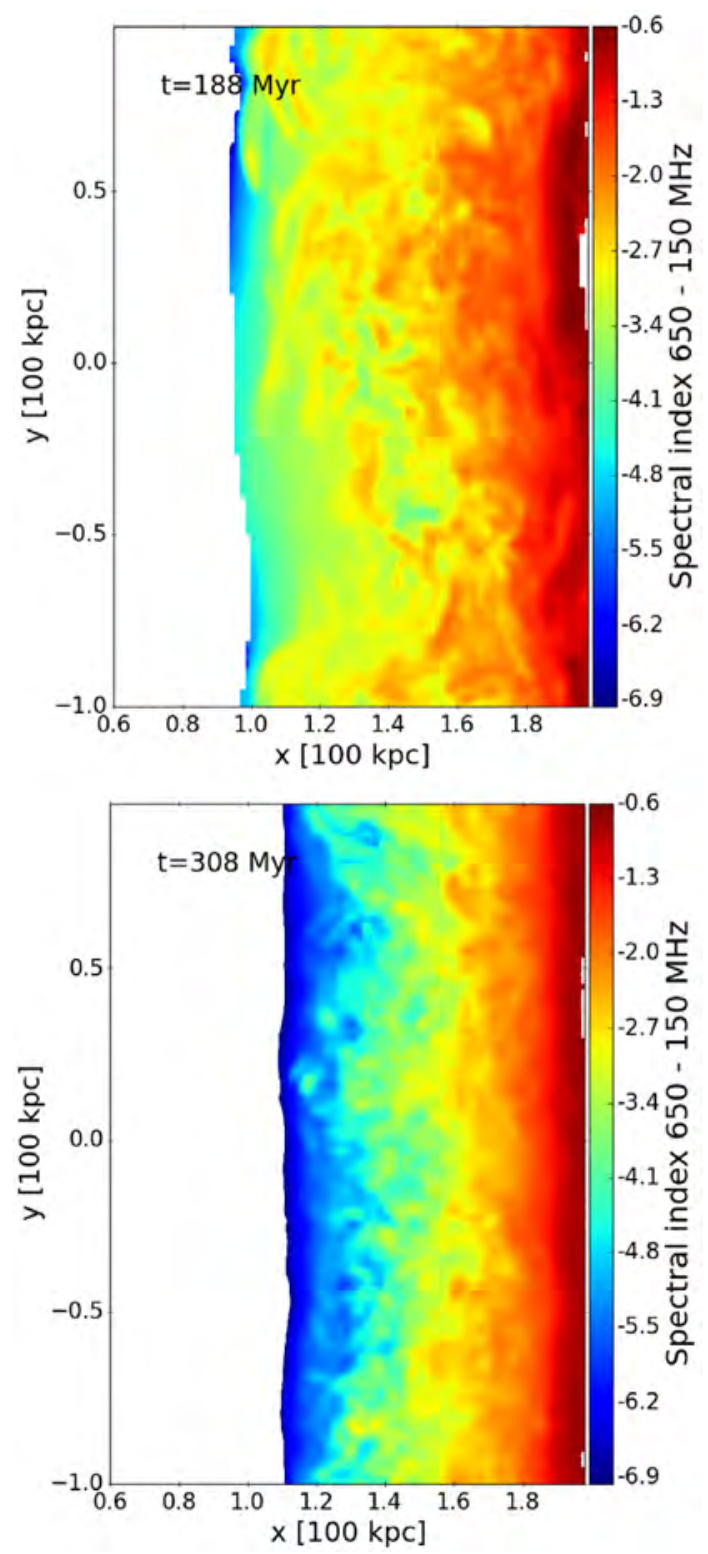

Figure 12. Spectral index maps obtained between 150 and $650 \mathrm{MHz}$ at $t_{\text {shock }}$ using equation (24). Top panel: $2 L / 3, \mathcal{M}=3$, and $\theta_{\mathrm{bn}}=0^{\circ}$ run. Bottom panel: $L / 4, \mathcal{M}=3$, and $\theta_{\mathrm{bn}}=0^{\circ}$.

that one should be careful when making use of it. One good way to confirm if it is applicable or not is by cross-checking with the result from high-resolution spectral index maps.

This has also been found in previous studies. For example, Kang (2015) showed that the relation in equation (26) only holds for planar shocks, but not for spherical shocks. In the presence of a turbulent medium, the geometry of the shock is more complicated leading to the evolution observed in Fig. 14.

We find that the integrated spectral index in Fig. 14 is biased towards higher Mach numbers for all runs. The main reason for this is that the brightest radio emitting regions correspond to the strongest shock compression regions, which in average gives a bias towards higher Mach numbers. In Section 4.4, we will show an analysis of the Mach number distribution and compare it to the one inferred from the thermal fluid. In Fig. 15, we show how the emission at the shock front correlates with the magnetic field strength in 3D and 2D. 

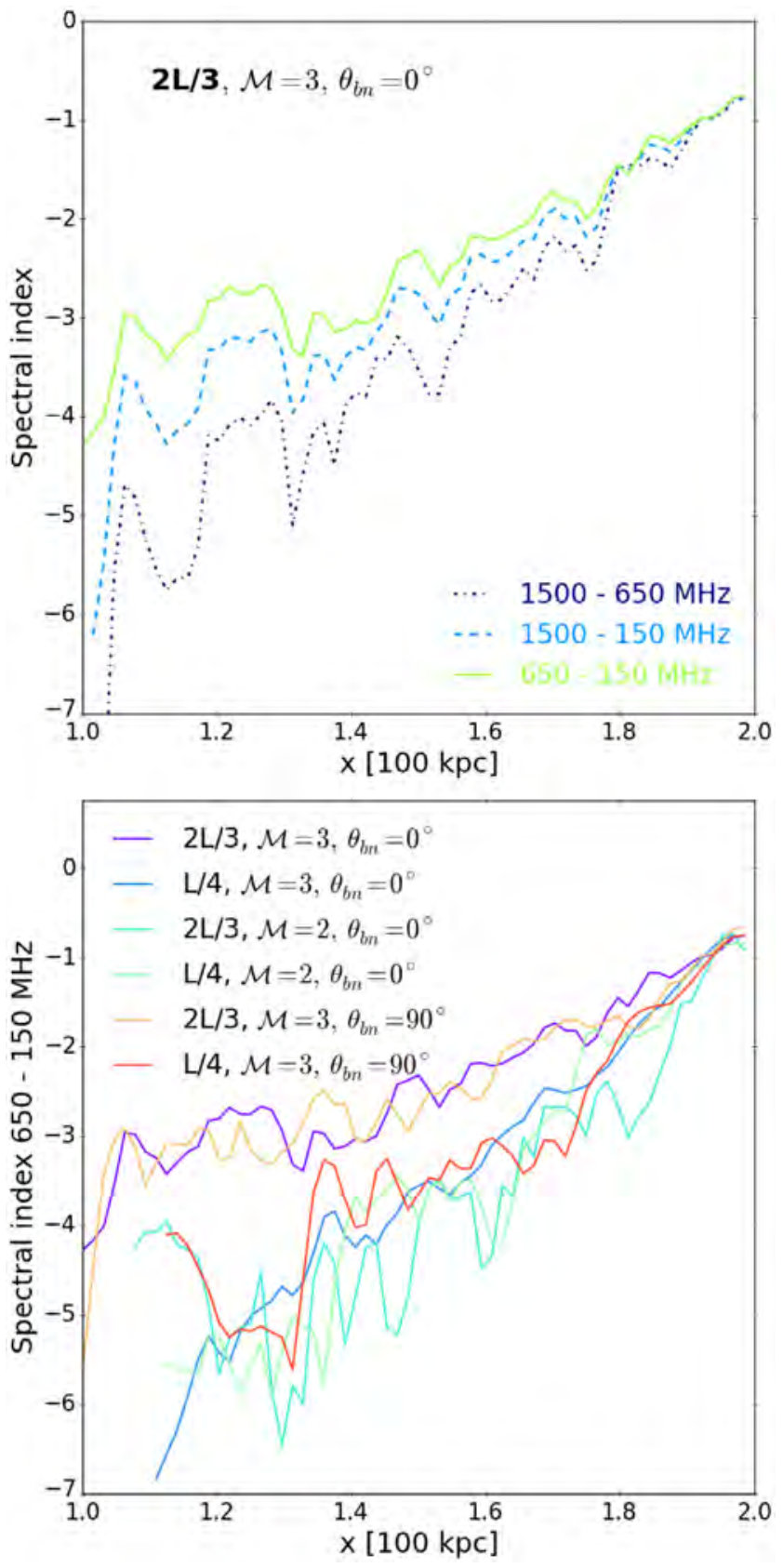

Figure 13. Top panel: Spectral index profile for the $2 L / 3, \mathcal{M}=3$, and $\theta_{\mathrm{bn}}=$ $0^{\circ}$ case at $t=178 \mathrm{Myr}$. Bottom panel: Spectral index profiles between 150 and $650 \mathrm{MHz}$ for all the cases. The profiles are computed at the same times as in Fig. 10.

We show the evolution for the $2 L / 3$ case with $\mathcal{M}=3$ and $\theta_{\mathrm{bn}}=0^{\circ}$ run. The emissivity computed from equation (20) scales with the magnetic field and frequency as

$\mathcal{J}_{\text {syn }} \propto B^{(p+1) / 2} v^{-(p-1) / 2}$

(see Engel 1979) where $\alpha=(p-1) / 2$ is the spectral index and $p$ is related to the Mach number through equations (13) and (12),

$\alpha=\frac{(p-1)}{2}=\frac{(q-3)}{2}=\frac{\mathcal{M}^{2}+3}{2\left(\mathcal{M}^{2}-1\right)}$.

In the top panel of Fig. 15, we show this relation for different Mach numbers with coloured lines and we add an additional black dashed

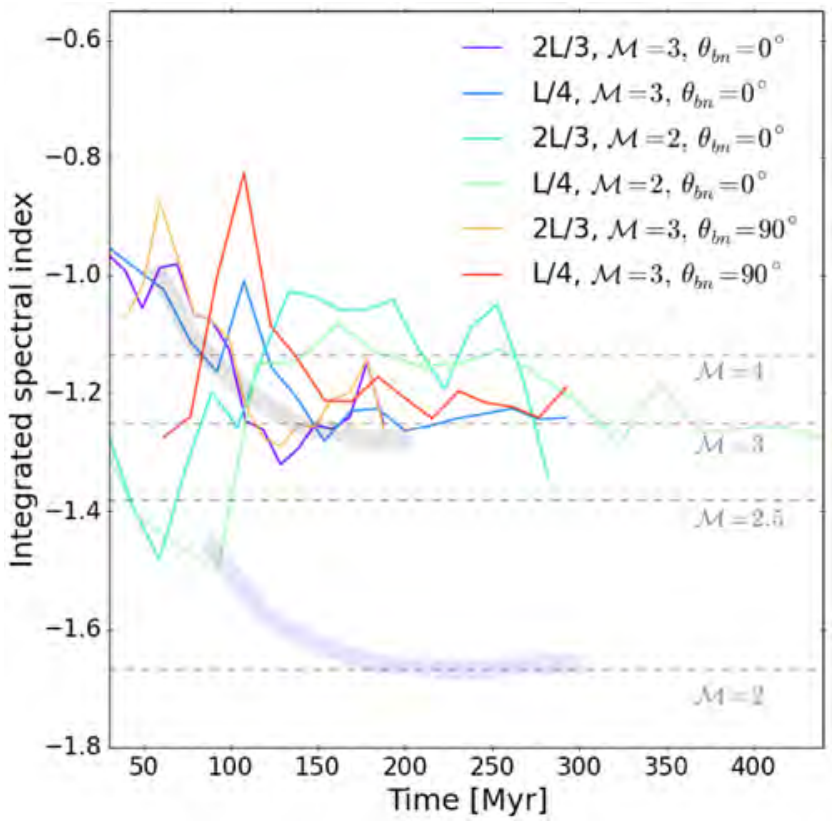

Figure 14. Integrated spectral index evolution computed from fitting the total flux. The grey horizontal dashed lines show the expected integrated spectral index assuming that $\alpha_{\text {int }}=\alpha+1 / 2$ for different Mach numbers (see equation 26). The shaded areas correspond to the uniform runs with $\mathcal{M}=2$ (blue) and $\mathcal{M}=3$ (grey) shocks.

line corresponding to a fit of all the data points. Overall, we find that there is always a systematic mismatch with the initial real Mach number of the shock. During the first $\sim 20 \mathrm{Myr}$, the 3D distribution shows a sharper relation coinciding with what is expected from equation (28). Nevertheless, shortly after that there will be a spread in the Mach number and magnetic field distribution along the shock front. This spread will keep changing as a consequence of all the turbulent motions in the medium. The black dashed line shows that the relation is biased towards larger Mach numbers. For the $2 \mathrm{D}$ case shown in the bottom panel of Fig. 15, we considered a magnetic field weighted with the radio emissivity:

$B_{w}=\frac{\int B \mathcal{J}_{\mathrm{syn}} \mathrm{d} Z}{\int \mathcal{J}_{\mathrm{syn}} \mathrm{d} Z}$

We show the same relation pointed out in the upper panel as a reference. The dashed black line in this case corresponds to the fit of all data points in the 2D map. While in this case we have less data points due to the integral along the LOS, it is interesting to see that the bias towards higher Mach numbers is still there. This suggests that the bias is not due to projection effects. We will further discuss the reason behind this bias in Section 4.4.

\subsection{Spectral properties}

\subsubsection{Magnetic field}

One important feature that characterizes the magnetic field is its power spectrum:

$P_{i j}(\boldsymbol{k})=\frac{1}{(2 \pi)^{3}} \iiint \mathrm{e}^{-i \boldsymbol{k} \cdot \boldsymbol{x}} R_{i j}(\boldsymbol{k}) \mathrm{d} \mathbf{k}$,

where $R_{i j}=\left\langle b_{i}\left(\boldsymbol{x}_{\mathbf{0}}\right) b_{j}\left(\boldsymbol{x}_{\mathbf{0}}+\boldsymbol{x}\right)\right\rangle$ is the two-point correlation function between the magnetic fields $b_{i}$ and $b_{j}$ (e.g. Batchelor 1951). In the case of homogeneous and isotropic fields, the relation between 


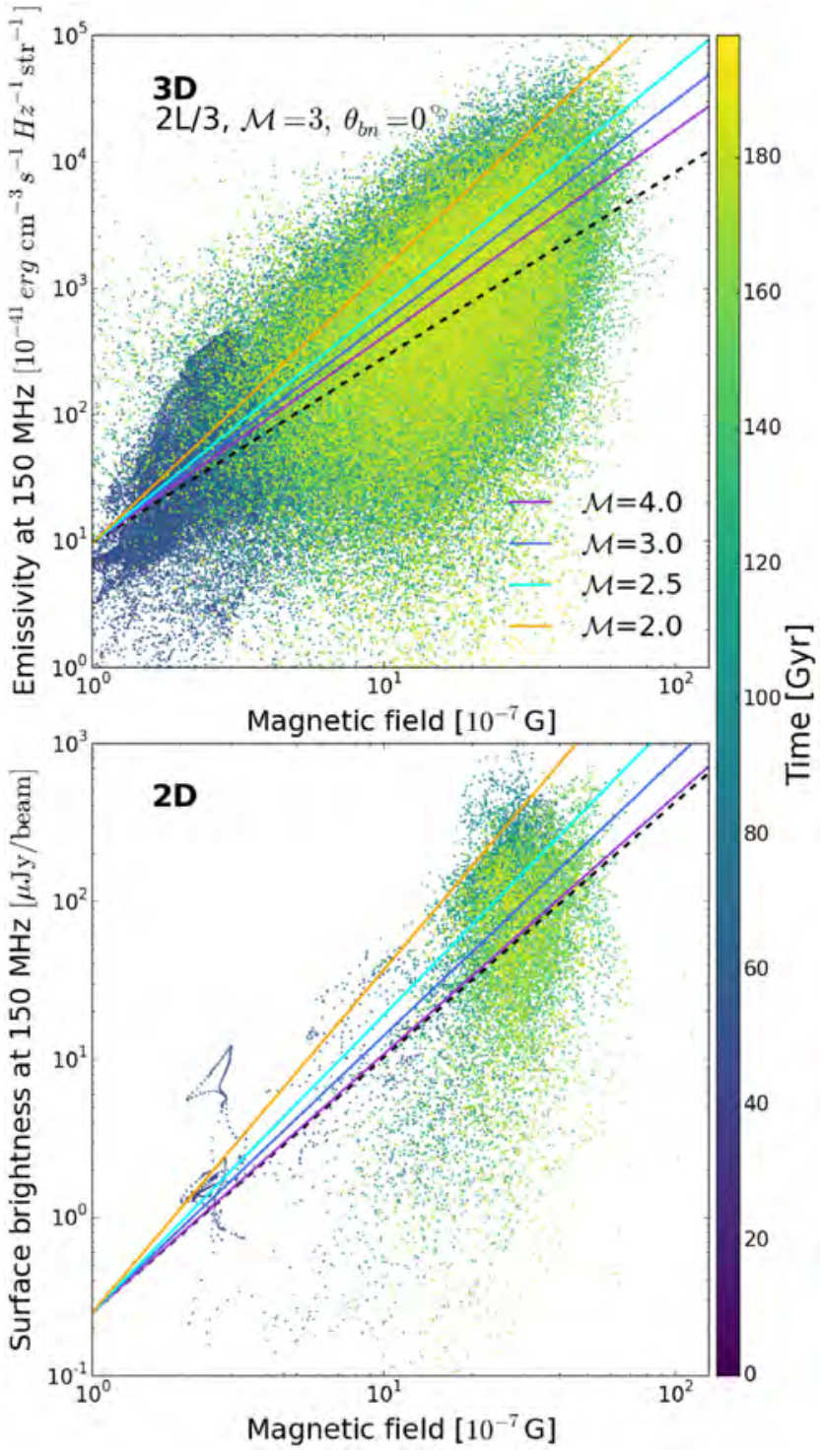

Figure 15. Phase plots of the magnetic field versus the radio emission at $150 \mathrm{MHz}$ at the cells where the shock front is located for the $2 L / 3, \mathcal{M}=3$, and $\theta_{\mathrm{bn}}=0^{\circ}$ run. Top panel: Values extracted out of the 3D distributions, i.e. the emissivity $\mathcal{J}_{\text {syn }}$. The coloured lines show the expected fit for different Mach numbers and the black dashed line shows a fit of the data at $t=$ 188 Myr. Bottom panel: Values extracted out of the 2D maps, i.e. here we have the surface brightness and the values of the magnetic field weighted with the emission (see equation 29).

the spectral energy and the 1D power spectrum is found to be

$E(k)=2 \pi k^{2} P_{i i}(k)$.

We will refer to the $1 \mathrm{D}$ power spectrum $P_{i i}(k)$ simply as $P(k)$. In order to obtain the $1 \mathrm{D}$ power spectrum, we averaged the $3 \mathrm{D}$ power spectrum of the magnetic field over spherical shells:

$P(k)=\frac{1}{N_{k}} \sum_{k-\frac{1}{2}<|\boldsymbol{k}| \leq k+\frac{1}{2}} P(\boldsymbol{k})$.

We computed the energy power spectrum in the right half part of the simulation (region III, [0, 200] kpc) (see Fig. 4). In the top panel of Fig. 16, we show the final magnetic energy spectra for all our runs and in the bottom panel we show the final power spectra computed for the
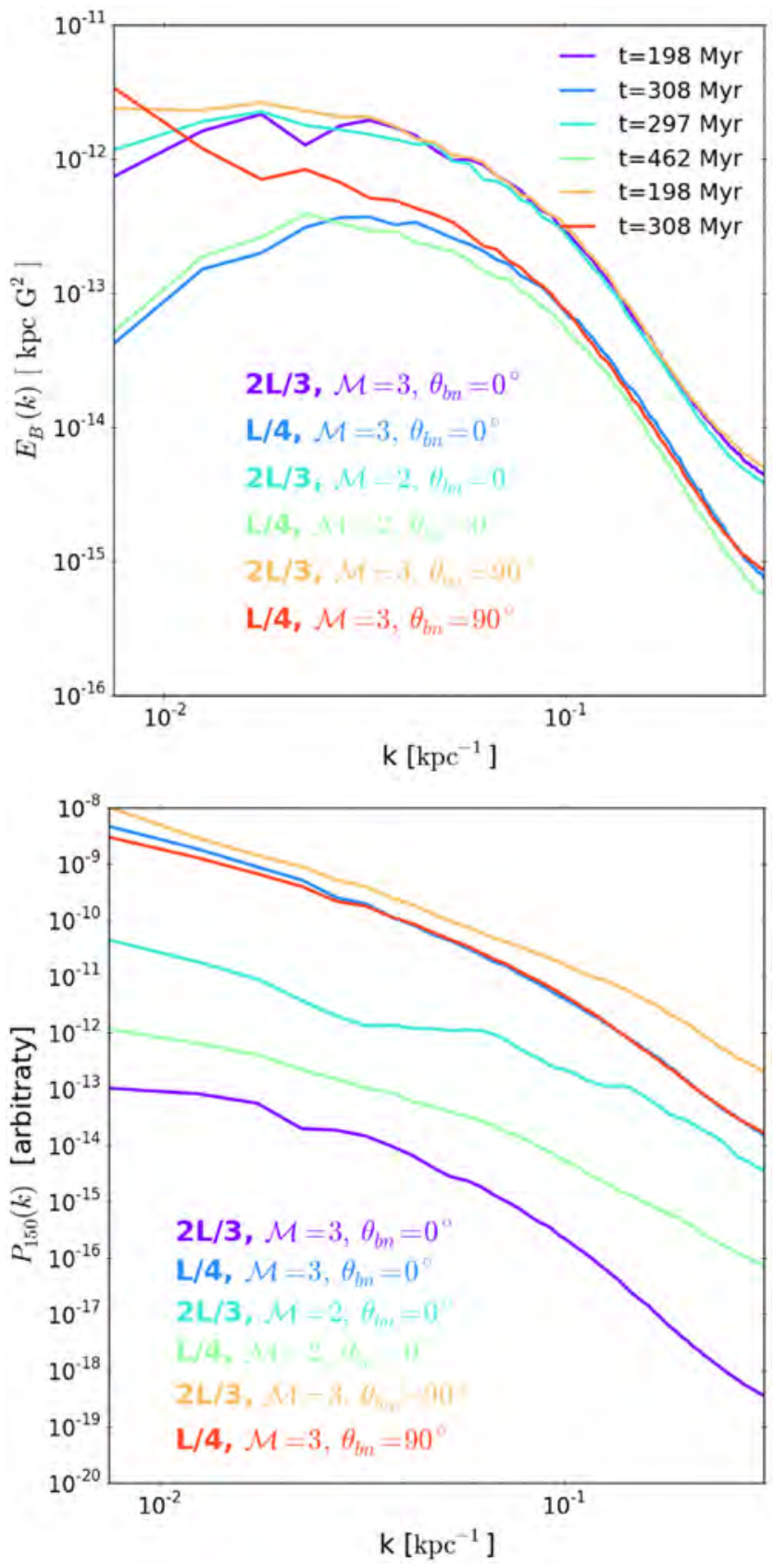

Figure 16. Top panel: Final magnetic energy spectrum for all our runs. The spectra are computed in a $(200 \mathrm{kpc})^{3}$ volume through equations (31) and (32). The final time-step differs for each run and it is specified in the legend. Bottom panel: Final power spectrum of the synchrotron emissivity at $150 \mathrm{MHz}$ for all our runs (same times as in the top panel).

emissivity at $150 \mathrm{MHz}$. In Fig. 17, we present the whole evolution of the magnetic energy spectrum $E_{B}(k)$ condensed in the form maps. Each of these maps contains the following information: the $y$-axis shows the evolution and the $x$-axis shows the wavenumber $k$ coloured with the amplitude of the magnetic energy spectrum at that $k$. In this way, the darker colours denote the regions where the power is peaking (see Fig. 16 as a reference). The dashed grey line is a reference for the reader to know when the shock enters this turbulent region, i.e. region III. We are interested in understanding the effect of shocks in the magnetic energy spectrum, in presence of an ICM with decaying turbulence. We can see in Fig. 17 two important features: (i) the 

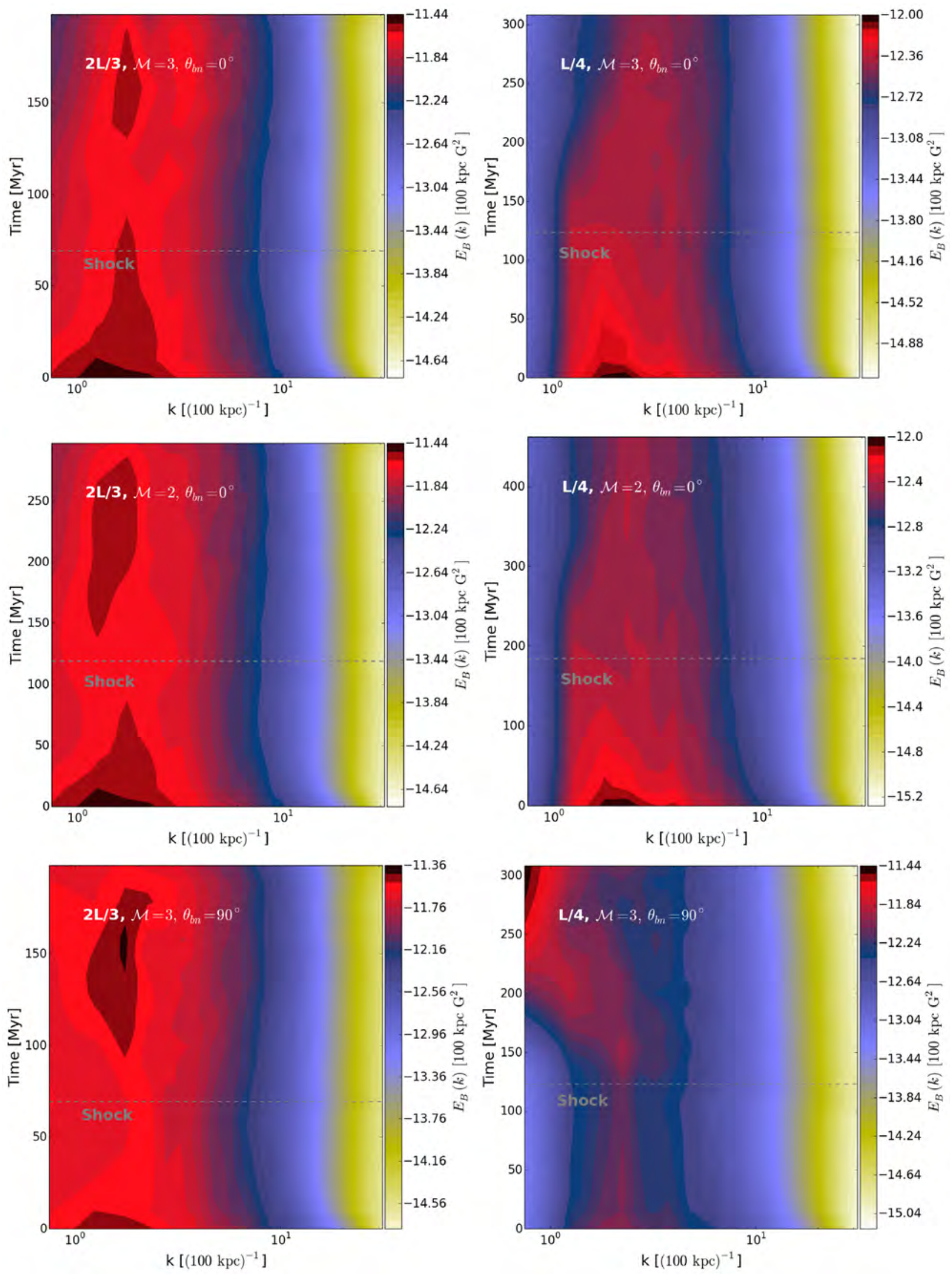

Figure 17. Evolution of the magnetic energy spectrum in the turbulent part of the box (region III [0,2] in Fig. 4 corresponding to a (200 kpc) $)^{3}$ cube). At each time-step ( $y$-axis), we show the magnetic power spectrum by colouring the $x$-axis with its amplitude (see colourbar in logarithmic scale). 
wavenumbers $k \gtrsim 10$ (corresponding to scales $\lesssim 10 \mathrm{kpc}$ ) are largely unaffected by shocks with strength $\mathcal{M}=2-3$. The resolution may play a role in this case. For example, when a shock enters a turbulent medium, Richtmyer-Meshkov instabilities peaking at small scales can arise. Nevertheless, such instability would take a long time to grow considering weak shocks and therefore we do not expect those to have a major effect in the context of radio relics; (ii) the shock compression has a notable effect only in the $\theta_{\mathrm{bn}}=90^{\circ}$ cases (see the two bottom panels of Fig. 17) at $k \lesssim 2$ (corresponding to scales $\gtrsim 50 \mathrm{kpc}$ ). In these cases, power shifts towards smaller wavenumbers (larger scales) due to the new turbulence introduced after the shock passage. This agrees with previous results from cosmological MHD simulations. In Domínguez-Fernández et al. (2019), we observed the same effect when analysing the evolution of a merging galaxy cluster over a time span of almost 10 Gyr. After every merger, shock waves are created and after every shock crossing, the magnetic power shifts to smaller wavenumbers (or larger scales).

\subsubsection{Emission}

In this subsection, we make the same analysis as in subsection 4.3.1, but for the radio emission and we compare it to the results from the magnetic field. We compute the power spectrum of the 3D distributions and also of the 2D maps (computed by integrating along the LOS). The $1 \mathrm{D}$ power spectrum is obtained by averaging the $3 \mathrm{D}$ spectrum over spherical shells (see equation 32) as mentioned above; while for the $2 \mathrm{D}$ maps, we averaged the $2 \mathrm{D}$ spectrum over annuli. Afterwards we can compute the characteristic length of the power spectrum for the emission as

$\lambda_{c}=\frac{\int k^{-1} P(k) \mathrm{d} k}{\int P(k) \mathrm{d} k}$,

and for the magnetic field as,

$\lambda_{B}=\frac{\int k^{-1} P_{B}(k) \mathrm{d} k}{\int P_{B}(k) \mathrm{d} k}$,

where $P(k)$ and $P_{B}(k)$ correspond to the power spectrum of the synchrotron emission (see equation 20) and the power spectrum of the magnetic field, respectively.

In the first two columns of Fig. 18, we show the results for the 3D case for all of the runs including also the characteristic scale of the magnetic field $\lambda_{B}$. The characteristic scale of the radio emission is in general of the same order of the characteristic scale of the magnetic field, that is of the order of $\lesssim 100 \mathrm{kpc}$. There are some specifics regarding (1) the Mach number: higher Mach numbers lead to larger emission scales, for example a $\mathcal{M}=3$ leads to a maximum scale of $\sim 100 \mathrm{kpc}$, whereas a $\mathcal{M}=2$ leads to a maximum of $\sim 80 \mathrm{kpc}$; and (2) the injection scale of the turbulence in the $\theta_{\text {bn }}=0^{\circ}$ case: the characteristic scale of the emission seems not to be affected by the injection scale, but it cannot be directly correlated with the magnetic field's scale. For example, the top panels of the first two columns in Fig. 18 show how $\lambda_{c}$ is of the same order for both cases while the underlying turbulence is different. In contrast, $\lambda_{B}$ is directly affected by the underlying turbulence and therefore it is of a different order. The type of injection scale of the turbulence in the $\theta_{\mathrm{bn}}=90^{\circ}$ case plays a big role for the evolution of $\lambda_{B}$, but this is not reflected in the evolution of $\lambda_{c}$. This happens only due to the fact that the acceleration efficiency, $\eta$, does not depend on $\theta_{\mathrm{bn}}$ in our modelling. The role of a varying $\eta$ will be subject of future work.

The characteristic scales of the integrated LOS variables are also shown in Fig. 18 (last two columns). In this case, $\lambda_{B}$ can be smaller than in reality is by roughly $17-23$ per cent in some cases, while $\lambda_{c}$ is only smaller by $\sim 10-15$ per cent. Therefore, we do not observe strong changes in these results due to projection effects.

In summary, we find that the characteristic scales that can be derived from the radio emission could serve as a good proxy for knowing the order of magnitude of the magnetic field's characteristic scale. However, we also find a rather complicated evolution that cannot give us a one-to-one correlation between these two scales and therefore this exercise alone will not give us much information regarding the type of turbulence existing in the outskirts of ICM. In practice, also the resolution of the radio telescopes plays an important role and will definitely affect these results.

\subsection{Mach number distributions}

\subsubsection{D distribution}

While the shock front's Mach number distribution peaks at its initial Mach number for almost the whole evolution, it develops a tail towards higher Mach numbers owing to turbulent motions. ${ }^{6}$ In order to study its impact on the emission, we obtain the emissivity, $\mathcal{J}_{\text {syn }}$, corresponding to the cells tracking the shock front at each time-step and correlated it with its Mach number. We can do this because the particles are being activated whenever they detect the shock front. In Fig. 19, we show the binned statistics for the two quantities obtained considering 16 time-steps

In order to generate an X-ray alike estimate of the Mach number, we computed the X-ray emissivity

$\mathcal{J}_{\text {X-ray }}=1.2 \times 10^{-28} T^{1 / 2}\left(\frac{\rho}{m_{p}}\right)^{2}$,

in units of erg $\mathrm{cm}^{-3} \mathrm{~s}^{-1} \mathrm{~Hz}^{-1} \mathrm{str}^{-1}$, where $m_{p}$ is the proton's mass and $T$ and $\rho$ are in cgs units. Thus, we selected the emission $\mathcal{J}_{\text {syn }}$ and $\mathcal{J}_{\mathrm{X} \text {-ray }}$ only at the shocked cells at each time-step in order to compute the binned statistics. We show the distributions at different times with dashed coloured lines and that of the whole evolution with the solid blue lines in Fig. 19. The top panels show the binned statistics considering the radio emissivity and the bottom panels show that of the X-ray emissivity.

The discrepancy between the two statistical distributions is evident. While the radio emissivity is always biased towards larger Mach numbers, the X-ray emissivity is biased towards smaller Mach numbers. In fact, the peak of the binned distribution of the radio emissivity (temporal envelope) is similar to the values in the tail of the real 3D Mach number distribution (i.e. see an example in Fig. B2 in Appendix B). Out of the six cases analysed, only the $L / 4, \mathcal{M}=2$, and $\theta_{\mathrm{bn}}=0^{\circ}$ case shows a partial match between both distributions. We also observe that the radio-Mach number statistics fluctuates with time such that the peak Mach number can vary a fair bit.

In addition, we analysed two runs with uniform media (all fields) but the same Mach numbers (i.e. $\mathcal{M}=2$ and $\mathcal{M}=3$ ). We verified that in this case the distributions of both emissivities and Mach numbers are the same. Hence, our results suggest that the difference in Mach numbers in radio and in X-ray is a result of turbulence.

In the presence of turbulence, the radio emissivity is not volumefilling, while the X-ray emissivity comes from the whole shock front. The fact that compression is different from region to region leads to a patchier radio emissivity that can probe a limited part of the shock front (see Fig. 9). Apart from that, the radio emission is biased towards higher Mach numbers because the initial CR energy

${ }^{6}$ See an example for the case $2 L / 3, \mathcal{M}=3$, and $\theta_{\text {bn }}=0^{\circ}$ in Fig. B2 of Appendix B. 
3D

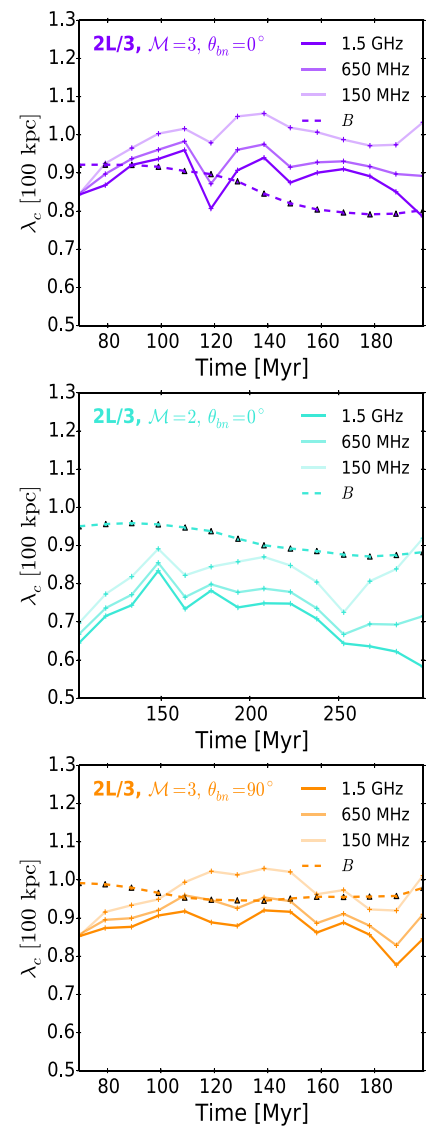

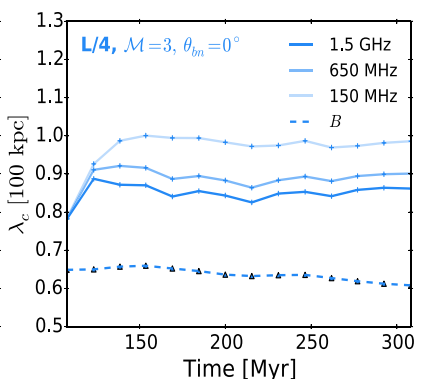
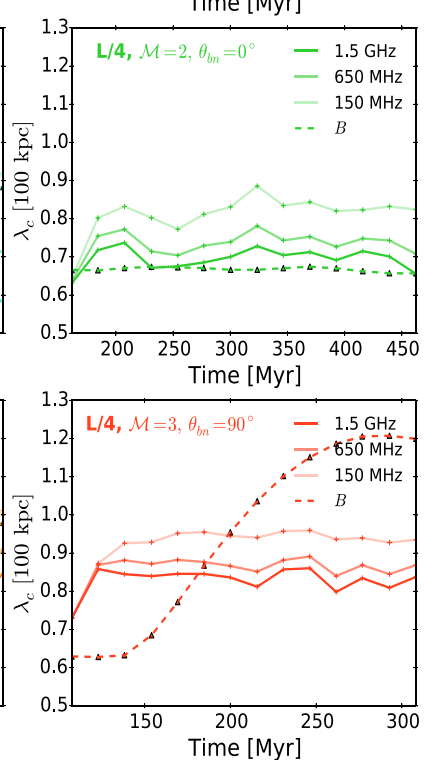

2D
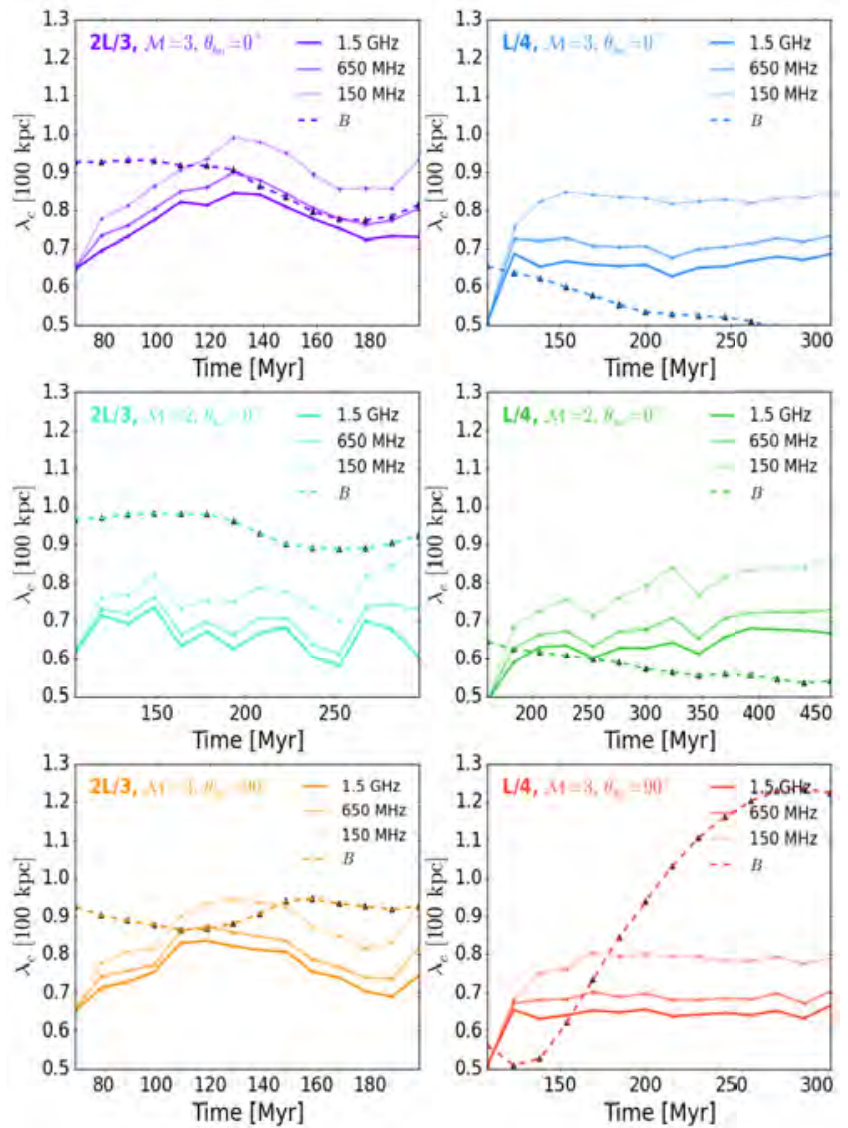

Figure 18. First two columns: Characteristic scale of the 3D distributions according to equation (33). Last two columns: Characteristic scale of the 2D distributions. Each panel shows the evolution of $\lambda_{c}$ for $1.5 \mathrm{GHz}, 650 \mathrm{MHz}$, and $150 \mathrm{MHz}$ and also the evolution of the magnetic field's characteristic scale $\lambda_{B}$.

is $\propto \mathcal{M}^{3}$ (which in our work corresponds to the normalization $N_{0}$ defined in equation 15). Finally, the magnetic field fluctuations created after the shock-crossing play an important role. As discussed in Section 4.1, the amplitude of the magnetic field fluctuations (which directly affects the radio emission) decreases more slowly than the velocity, density, and temperature fluctuations. This adds to the discrepancy because the X-ray emissivity depends only on the temperature and density fields.

\section{SUMMARY AND CONCLUSIONS}

We have presented a hybrid framework to compute the synchrotron emission from a shock wave propagating through a medium with decaying turbulence representing a small fraction of the ICM. In our framework, the MHD grid represents a thermal fluid, whereas Lagrangian particles represent $\mathrm{CR}$ electrons. We injected $\mathrm{CR}$ electrons at the shock discontinuity assuming DSA. Each CR electron evolves according to the $\mathrm{CR}$ transport equation in the diffusion approximation.

Our simulations explored shocks with Mach numbers characteristic of radio relics, i.e. $\mathcal{M}=2$ and $\mathcal{M}=3$. Moreover, we varied the downstream turbulence using turbulence-in-a-box simulations: a solenoidal subsonic turbulence with power peaking at $2 / 3$ of the box (case $2 L / 3$ ) and a solenoidal subsonic turbulence with power peaking at $1 / 4$ of the box (case $L / 4$ ). One snapshot of each simulation was selected as an initial condition for our shock-tube simulation. Our results can be summarized as follows:

(i) Impact of a shock on decaying turbulence: We find that mild shocks produce magnetic fluctuations in the downstream region that do not correlate with fluctuations in velocity, density and temperature. In fact, we find that magnetic fluctuations can increase even when velocity fluctuations decrease. This, in turn, can affect the final extent of the magnetic downstream. We find the strongest effect in perpendicular shocks, as expected from theory. Shocks with $\mathcal{M}=2$ travelling in a medium with smaller fluctuations, such as our $L / 4$ case, cause the least effect and seem to hardly modify the initial magnetic field distribution.

(ii) Radio emission: The existence of substructure in the synchrotron emission is a direct consequence of a turbulent medium. We found that $\mathcal{M}=2$ shocks in our set-up are unlikely to reproduce observable radio relics. The physical reason behind this is that $\mathcal{M}=2$ shocks are not strong enough to modify the initial pre-shock magnetic field. For example, the relic at Abell 2744 (e.g. Govoni et al. 2001; Eckert et al. 2016; Pearce et al. 2017; Paul et al. 2019) reaches a surface brightness of the order of tens of $\mathrm{mJy} \mathrm{beam}^{-1}$ at $1.4 \mathrm{GHz}$, which would require an acceleration efficiency of $\eta \sim 1$ in our $L / 4$ turbulence.

(iii) Discrepancies in the spectral index: Our spectral index profiles suggest that in the case of $\mathcal{M}=3$ shocks, a turbulent injection scale of $2 L / 3$ or even larger reproduces observations better 

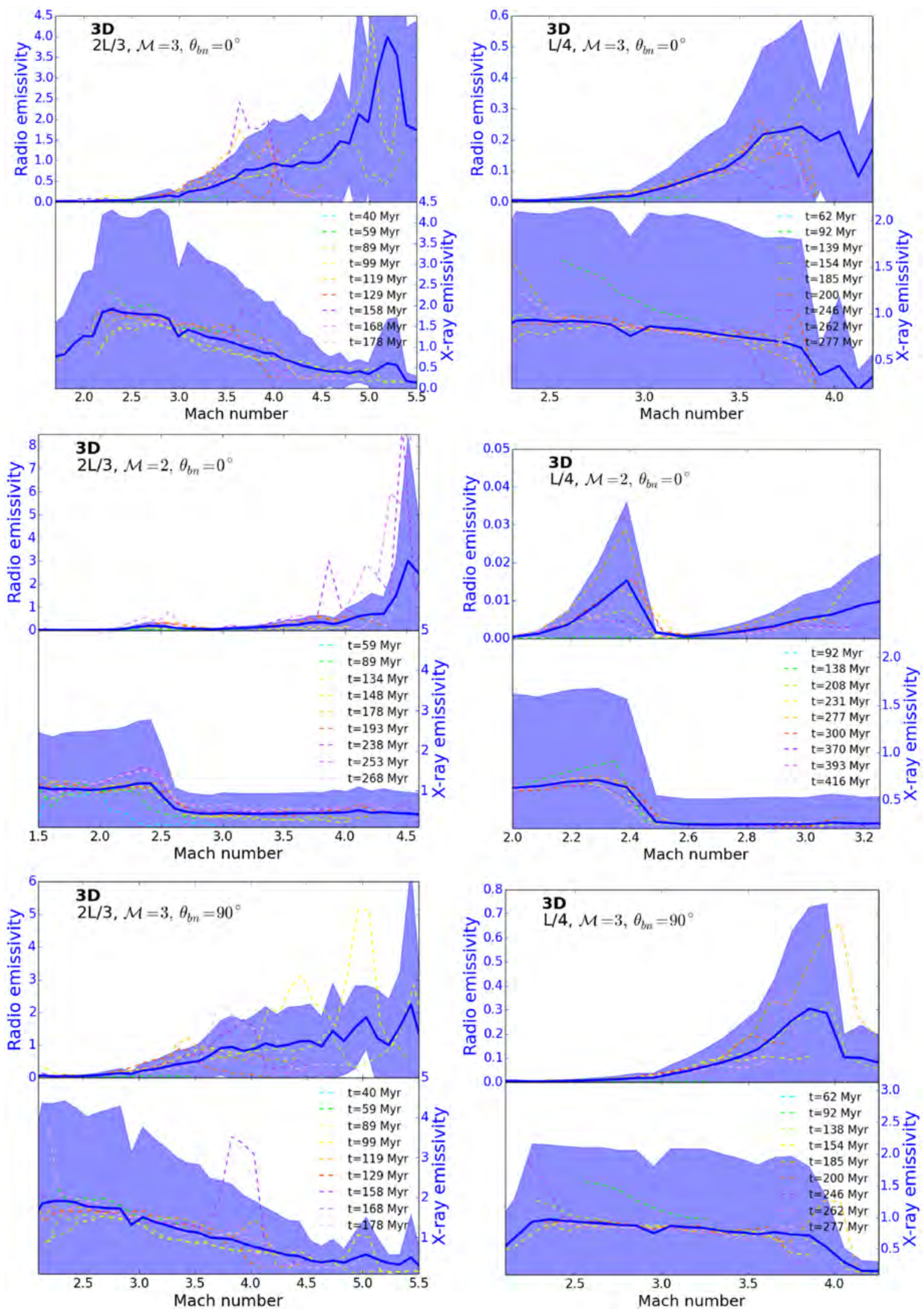

Figure 19. Binned statistics of radio emissivity at $1.5 \mathrm{GHz}$ (left axis) and X-ray emissivity (right axis) with the Mach number of the shock front for different time-steps (dashed coloured lines). The dark blue line shows the statistics taken into account the whole evolution and the shadowed areas denote the corresponding 75th percentile of the distribution. The emissivity is shown in units of $10^{-38} \mathrm{erg} \mathrm{cm}^{-3} \mathrm{~s}^{-1} \mathrm{~Hz}^{-1} \mathrm{str}^{-1}$. 
than the $L / 4$ case. The $L / 4$ initial magnetic field distribution allows for higher values of the magnetic field strength reflected in the tail of the PDF which steepens the spectral index profiles more than in the $2 L / 3$ case. We conclude that an initial turbulent, magnetic field distribution in the ICM must have a standard deviation smaller than $\sigma_{B}=1 \mu \mathrm{G}$. We compare our results of the integrated spectral index to the relation $\alpha_{\text {int }}=\alpha+1 / 2$ and find that this relation does not seem to hold in the presence of a turbulent medium. The reason for this is the distribution of Mach numbers within a shock front in a turbulent medium. As a consequence, the injected electrons will have different initial energy spectra.

(iv) Discrepancies in Mach numbers: We find that the synchrotron emission is biased towards larger Mach numbers when comparing to the X-ray emission. This agrees with previous numerical work (e.g. Hong et al. 2015) and a number of observations of radio relics. For example, X-ray observations of the Toothbrush relic in the cluster 1RXS J0603.3+4214 infer a Mach number of $\mathcal{M} \sim 1.5$ (e.g. Ogrean et al. 2013; van Weeren et al. 2016), while radio observations infer a higher Mach number of $\mathcal{M} \sim 3.7$ (e.g. Rajpurohit et al. 2018, 2020). The source of this discrepancy lies in (1) the stronger dependence of the synchrotron emission on the compression in the shock and (2) the fact that the amplitude of the magnetic field fluctuations (which affect the radio emission) decreases more slowly than the density and temperature fluctuations. Hence, higher Mach numbers in the tail of the Mach number distribution bias the overall Mach number.

(v) Magnetic energy spectrum: We find that scales $\lesssim 10 \mathrm{kpc}$ are largely unaffected by shocks with $\mathcal{M}=2-3$, independent of the type of turbulence. We find that the power shifts towards smaller wave numbers (larger scales) after shock passage which is more pronounced in perpendicular shocks.

(vi) Characteristic length of the radio emission: The characteristic lengths derived from the power spectrum of the emission, $\lambda_{c}$, and magnetic field, $\lambda_{B}$, are of the same order. We find that $\lambda_{c}$ is in general of the order of $\lesssim 100 \mathrm{kpc}$. Analysing the LOS variables, we do not observe strong projection effects and $\lambda_{B}$ and $\lambda_{c}$ are only $17-23$ per cent and $10-15$ per cent smaller than in $3 \mathrm{D}$.

In summary, we could identify the most important features that link the observable properties of radio relics with the dynamical properties of the upstream ICM. Our work confirms that the Mach numbers inferred from the radio emission are likely to be overestimates of the real Mach number of the thermal fluid in the presence of turbulence. This has been previously pointed out as a possible solution that can alleviate the problem of acceleration efficiencies and as a possible explanation for the non-detection of $\gamma$-rays from galaxy clusters (see Ackermann et al. 2010, 2014, 2016; Vazza \& Brüggen 2014). While CRe and CRp are expected to be accelerated at the same places, their acceleration mechanisms and efficiencies will differ (e.g. Caprioli \& Spitkovsky 2014a). CRe are accelerated preferentially at quasi-perpendicular shocks and CRp at quasi-parallel shocks. Recent works by Wittor et al. (2020) and Banfi, Vazza \& Wittor (2020) used cosmological MHD simulations to show that indeed the predominance of quasi-perpendicular shocks in merger and accretion shocks might be enough to explain the absence of CRp. Therefore, in future work we will include the obliquity dependence in our acceleration efficiency, $\eta$. Moreover, we intend to survey a larger range of possible parameters, both, for the ICM conditions and for the shock properties. This will help us to assess whether the large variety of relic sources can be explained by the model adopted here. We will also present a detailed study considering polarization ( $Q$ and $U$ Stokes parameters, as well as rotation measure), different lines of sight, projection, and beam effects.

\section{ACKNOWLEDGEMENTS}

The analysis presented in this work made use of computational resources on the JUWELS cluster at the Juelich Supercomputing Centre (JSC), under project 'stressicm' with FV as principal investigator and PDF as co-principal investigator. The $3 \mathrm{D}$ visualization of the synchrotron emissivity was done with the VISIT software (see Childs et al. 2012).

PDF, FV, and MB acknowledge the financial support from the European Union's Horizon 2020 program under the ERC Starting Grant 'MAGCOW', no. 714196. WEBB acknowledges the financial support from the Deutsche Forschungsgemeinschaft (DFG) via grant BR2026125.

We acknowledge our anonymous reviewer for helpful comments on the first version of this manuscript. Finally, we thank D. Ryu, C. Federrath, and R. Mohapatra for fruitful scientific discussions.

\section{DATA AVAILABILITY STATEMENT}

The data underlying this article will be shared on reasonable request to the corresponding author. Any links or identifiers are present in this article.

\section{REFERENCES}

Ackermann M. et al., 2010, ApJ, 717, L71

Ackermann M. et al., 2014, ApJ, 787, 18

Ackermann M. et al., 2016, ApJ, 819, 149

Banda-Barragán W. E., Federrath C., Crocker R. M., Bicknell G. V., 2018, MNRAS, 473, 3454

Banfi S., Vazza F., Wittor D., 2020, MNRAS, 496, 3648

Batchelor G. K., 1951, Math. Proc. Camb. Phil. Soc., 47, 359

Birdsall C. K. N., Estacio E. T., Plasma Theory and Simulation Group (PTSG), 2004, Comput. Phys. Commun., 164, 189

Blandford R., Eichler D., 1987, Phys. Rep., 154, 1

Blandford R. D., Ostriker J. P., 1978, ApJ, 221, L29

Bonafede A. et al., 2018, MNRAS, 478, 2927

Böttcher M., Dermer C. D., 2010, ApJ, 711, 445

Botteon A., Brunetti G., Ryu D., Roh S., 2020, A\&A, 634, A64

Brüggen M., Bykov A., Ryu D., Röttgering H., 2012, Space Sci. Rev., 166 , 187

Brunetti G., Jones T. W., 2014, Int. J. Mod. Phys. D, 23, 1430007

Bykov A. M., Kaastra J. S., Brüggen M., Markevitch M., Falanga M., Paerels F. B. S., 2019, Space Sci. Rev., 215, 27

Calder A. C. et al., 2002, ApJS, 143, 201

Caprioli D., Spitkovsky A., 2014a, ApJ, 783, 91

Caprioli D., Spitkovsky A., 2014b, ApJ, 794, 46

Childs H. et al., 2012, High Performance Visualization-Enabling ExtremeScale Scientific Insight, Chapman and Hall/CRC, Boca Raton, FL, p. 357

Clarke T. E., Ensslin T. A., 2006, AJ, 131, 2900

Dedner A., Kemm F., Kröner D., Munz C. D., Schnitzer T., Wesenberg M., 2002, J. Comput. Phys., 175, 645

Di Gennaro G. et al., 2018, ApJ, 865, 24

Domínguez-Fernández P., Vazza F., Brüggen M., Brunetti G., 2019, MNRAS, 486, 623

Donnert J., Vazza F., Brüggen M., ZuHone J., 2018, Space Sci. Rev., 214, 122

Drury L. O., 1983, Rep. Prog. Phys., 46, 973

Eckert D., Jauzac M., Vazza F., Owers M., Kneib J.-P., Tchernin C., Intema H., Knowles K., 2016, MNRAS, 461, 1302

Egan H., O'Shea B. W., Hallman E., Burns J., Xu H., Collins D., Li H., Norman M. L., 2016, preprint (arXiv:1601.05083)

Engel A. R., 1979, Phys. Bull., 30, 158

Eswaran V., Pope S. B., 1988, Phys. Fluids, 31, 506

Federrath C., Roman-Duval J., Klessen R. S., Schmidt W., Mac Low M. M., 2010, A\&A, 512, A81 
Fryxell B. et al., 2000, ApJS, 131, 273

Ginzburg V. L., Syrovatskii S. I., 1965, ARA\&A, 3, 297

Govoni F., Feretti L., Giovannini G., Böhringer H., Reiprich T. H., Murgia M., 2001, A\&A, 376, 803

Guo X., Sironi L., Narayan R., 2014, ApJ, 797, 47

Heavens A. F., Meisenheimer K., 1987, MNRAS, 225, 335

Hong S. E., Kang H., Ryu D., 2015, ApJ, 812, 49

Kang H., 2015, J. Korean Astron. Soc., 48, 9

Kang H., Ryu D., Jones T. W., 2012, ApJ, 756, 97

Kang H., Ryu D., Jones T. W., 2017, ApJ, 840, 42

Kang H., Ryu D., Ha J.-H., 2019, ApJ, 876, 79

Kardashev N. S., 1962, AZh, 39, 393

Kritsuk A. G. et al., 2011, ApJ, 737, 13

Landau L. D., Lifshitz E. M., eds, 1987, Fluid Mechanics, Course of Theoretical Physics, Vol. 6, 2nd edn. Butterworth-Heinemann, Oxford

Lee D., Deane A. E., Federrath C., 2009, in Pogorelov N. V., Audit E., Colella P., Zank G. P., eds, ASP Conf. Ser. Vol. 406, A New Multidimensional Unsplit MHD Solver in FLASH3. Astron. Soc. Pac., San Francisco, p. 243

Locatelli N. T. et al., 2020, MNRAS, 496, L48

Mignone A., Bodo G., Massaglia S., Matsakos T., Tesileanu O., Zanni C., Ferrari A., 2007, ApJS, 170, 228

Mimica P., Aloy M. A., 2012, MNRAS, 421, 2635

Miniati F., 2014, ApJ, 782, 21

Miyoshi T., Kusano K., 2005, J. Comput. Phys., 208, 315

Nuza S. E., Gelszinnis J., Hoeft M., Yepes G., 2017, MNRAS, 470, 240

Ogrean G. A., Brüggen M., van Weeren R. J., Röttgering H., Croston J. H., Hoeft M., 2013, MNRAS, 433, 812

Owen F. N., Rudnick L., Eilek J., Rau U., Bhatnagar S., Kogan L., 2014, ApJ, 794, 24

Park J., Caprioli D., Spitkovsky A., 2015, Phys. Rev. Lett., 114, 085003

Paul S., Salunkhe S., Datta A., Intema H. T., 2019, MNRAS, 489, 446

Pearce C. J. J. et al., 2017, ApJ, 845, 81

Pinzke A., Oh S. P., Pfrommer C., 2013, MNRAS, 435, 1061

Rajpurohit K. et al., 2018, ApJ, 852, 65

Rajpurohit K. et al., 2020, A\&A, 636, A30

Ryu D., Kang H., Hallman E., Jones T. W., 2003, ApJ, 593, 599

Ryu D., Kang H., Cho J., Das S., 2008, Science, 320, 909

Ryu D., Kang H., Ha J.-H., 2019, ApJ, 883, 60

Schaal K., Springel V., 2015, MNRAS, 446, 3992

Schmidt W., Niemeyer J. C., Hillebrandt W., 2006, A\&A, 450, 265

Sironi L., Spitkovsky A., Arons J., 2013, ApJ, 771, 54

Skillman S. W., Xu H., Hallman E. J., O'Shea B. W., Burns J. O., Li H., Collins D. C., Norman M. L., 2013, ApJ, 765, 21

Vaidya B., Mignone A., Bodo G., Rossi P., Massaglia S., 2018, ApJ, 865, 144

van Weeren R. J., Röttgering H. J. A., Brüggen M., Hoeft M., 2010, Science, 330,347

van Weeren R. J., Brüggen M., Röttgering H. J. A., Hoeft M., 2011, JA\&A, 32,505

van Weeren R. J., Röttgering H. J. A., Intema H. T., Rudnick L., Brüggen M., Hoeft M., Oonk J. B. R., 2012, A\&A, 546, A124

van Weeren R. J. et al., 2016, ApJ, 818, 204

van Weeren R. J. et al., 2017, ApJ, 835, 197

van Weeren R. J., de Gasperin F., Akamatsu H., Brüggen M., Feretti L., Kang H., Stroe A., Zandanel F., 2019, Space Sci. Rev., 215, 16

Vazza F., Brüggen M., 2014, MNRAS, 437, 2291

Vazza F., Dolag K., Ryu D., Brunetti G., Gheller C., Kang H., Pfrommer C., 2011, MNRAS, 418, 960

Vazza F., Jones T. W., Brüggen M., Brunetti G., Gheller C., Porter D., Ryu D., 2017, MNRAS, 464, 210

Vazza F., Brunetti G., Brüggen M., Bonafede A., 2018, MNRAS, 474, 1672

Wittor D., Hoeft M., Vazza F., Brüggen M., Domínguez-Fernández P., 2019, MNRAS, 490, 3987

Wittor D., Vazza F., Ryu D., Kang H., 2020, MNRAS, 495, L112

\section{SUPPORTING INFORMATION}

Supplementary data are available at MNRAS online.

emiss5.gif

Please note: Oxford University Press is not responsible for the content or functionality of any supporting materials supplied by the authors. Any queries (other than missing material) should be directed to the corresponding author for the article.

\section{APPENDIX A: NUMERICAL DIVERGENCE OF THE MAGNETIC FIELD}

The $\nabla \cdot \boldsymbol{B}=0$ condition in the particle module of the PLUTO code is maintained with the hyperbolic divergence cleaning technique where the induction equation is coupled to a GLM (e.g. Dedner et al. 2002). In Kritsuk et al. (2011), it has been argued that the best results for the divergence-free evolution of the magnetic field are achieved using the CT method. Keeping this in mind, we tested the differences of the $\nabla \cdot \boldsymbol{B}=0$ condition with the GLM and CT techniques. In Fig. A1, we show the evolution of the magnetic field divergence using the same set-up as our $2 L / 3, \mathcal{M}=3$, and $\theta_{\mathrm{bn}}=90^{\circ}$ run (see Table 1 ) for the whole simulation box. Both methods are comparable and keep the numerical magnetic field divergence under $\sim 0.001$ per cent of the local magnetic field value. This demonstrates that the use of the GLM divergence cleaning technique is robust against CT for our particular set-up. Thus, we do not expect our numerical scheme to have an impact in our final synchrotron emission maps. Additionally, we show how the divergence condition behaves for the interface between regions II and III in our setup in Fig. A1. The effects of the initial interpolation of the external input get diminished after a few steps and the numerical magnetic field divergence drops again below $\sim 0.001$ per cent before the shock enters the region of interest (i.e.

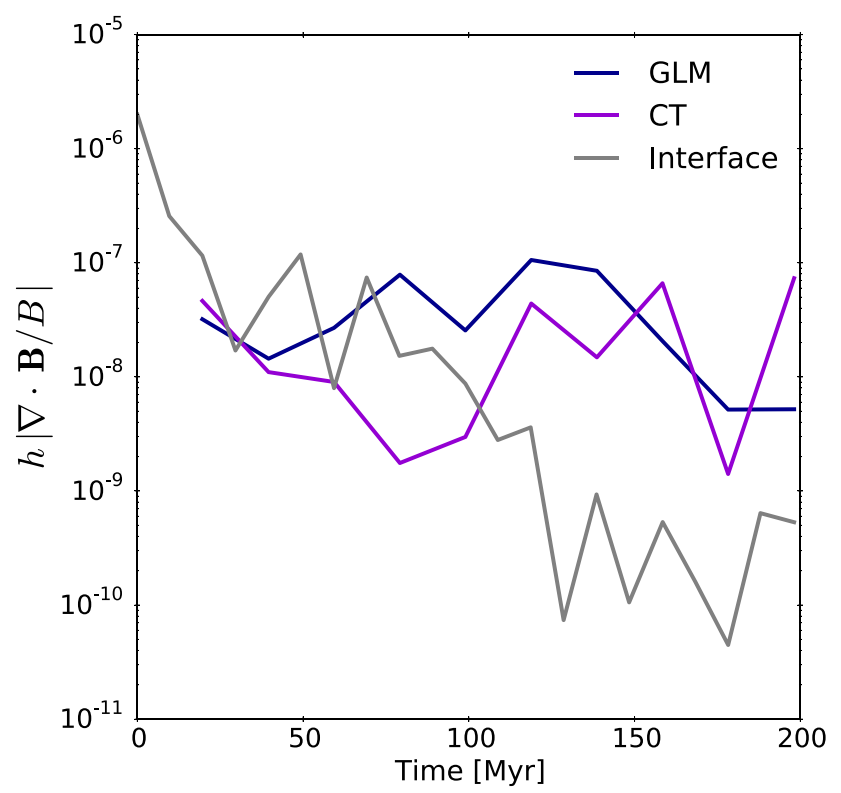

Figure A1. Test on the numerical conservation of the $\nabla \cdot \boldsymbol{B}=0$ condition (where $h=\Delta x$ ). We compare two runs only differing in their divergence cleaning method: GLM (blue) and CT (purple). The grey line shows the corresponding evolution for the interface between regions II and III using the GLM method. We performed this test using the set-up $2 L / 3, \mathcal{M}=3$, and $\theta_{\mathrm{bn}}=90^{\circ}$. 
region III); see an additional discussion about this in appendix D of Banda-Barragán et al. (2018).

\section{APPENDIX B : SHOCK FINDER}

The algorithm to find shocks is already implemented in the PLUTO code (see Vaidya et al. 2018). In the first step, shock cells are tagged through the $\nabla \cdot \boldsymbol{v}<0$ condition. Then we implemented an extra condition for the activation time of the tracer particles in order to compute the initial Mach number and compression ratio. This in turn is needed for assigning the initial energy spectra with an spectral index $q$ (see equation 12) to each tracer particle. The Mach number at the shock centre is computed from the Rankine-Hugoniot pressure

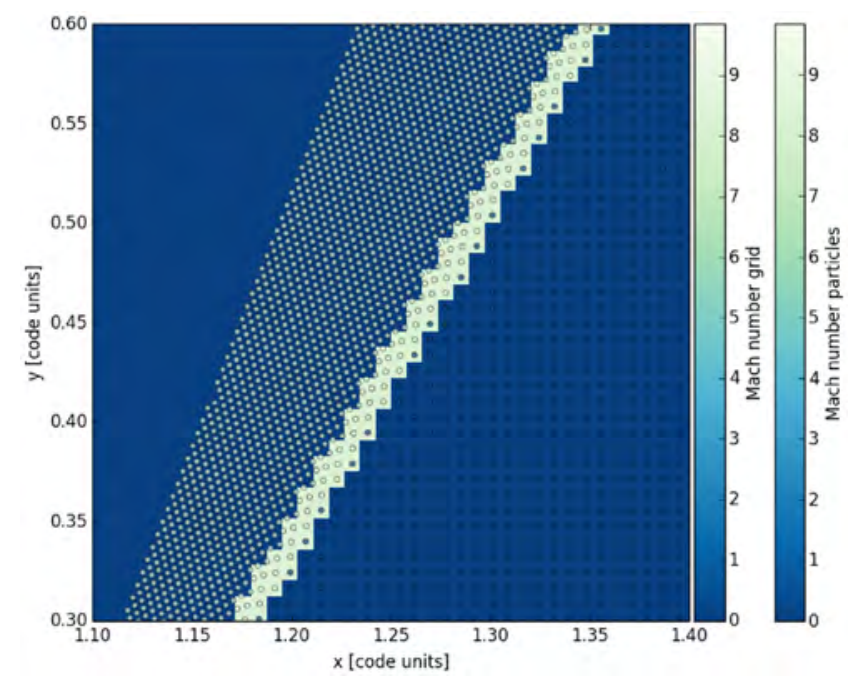

Figure B1. Numerical test on the shock's directionality. We show a 2D parallel shock propagating with an angle of $60^{\circ}$.

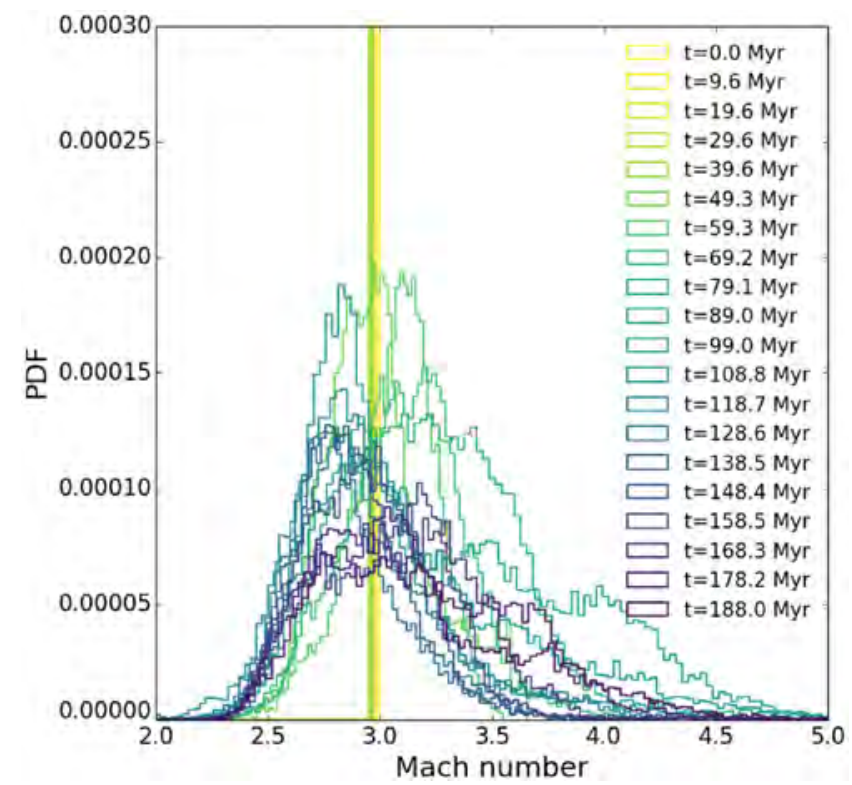

Figure B2. Evolution of Mach number distribution at the shock front for the case $2 L / 3, \mathcal{M}=3$, and $\theta_{\mathrm{bn}}=0^{\circ}$. jump condition:

$\Delta \log p \geq\left.\log \frac{p_{2}}{p_{1}}\right|_{\mathcal{M}=\mathcal{M}_{\min }}$,

where the subscripts 1 and 2 denote the pre-shock and postshock regions, respectively. The minimum Mach number is set to $\mathcal{M}_{\text {min }}=1.3$ and it acts as a threshold to filter out weaker shocks. The pressure jump is computed with the neighbouring cells along the three directions for which a Mach number distribution is finally obtained: $\mathcal{M}^{2}=\mathcal{M}_{x}^{2}+\mathcal{M}_{y}^{2}+\mathcal{M}_{z}^{2}$ (see Ryu et al. 2003; Vazza et al. 2011; Schaal \& Springel 2015).

The PLUTO code is able to compute the compression ratio for the update of the spectra once the particle has crossed a shock, nevertheless we implemented this extra condition as it was necessary for computing the compression ratio at the time of activation and not a time-step afterwards as in Vaidya et al. (2018). In this fashion, the tracer particles have an initial spectrum consistent with DSA theory at the time of activation and after a time-step their spectra will evolve subject to radiative losses.

We tested the directionality of the shock finder by setting up a 2D parallel shock propagating with an angle of $60^{\circ}$ with respect to the $x$-axis and placing one particle per cell on a quarter of the domain. The shock propagates with a Mach number of $\mathcal{M}=10$ and the interpolation of the grid quantity at the particle position in PLUTO is done with standard techniques used for PIC codes (see Birdsall et al. 2004). We used the nearest grid point method for the implementation of this test. In Fig. B1, we show the Mach grid distribution as well as the particle's interpolated Mach number for a snapshot of this set-up. We also show the evolution of the shock's Mach number in our final set-up for one of our studied cases in Fig. B2.

\section{APPENDIX C: RESOLUTION}

We tested our set-up doubling the resolution to $512 \times 256 \times 256$ cells and number of Lagrangian particles to 25165824 . Here, we will limit

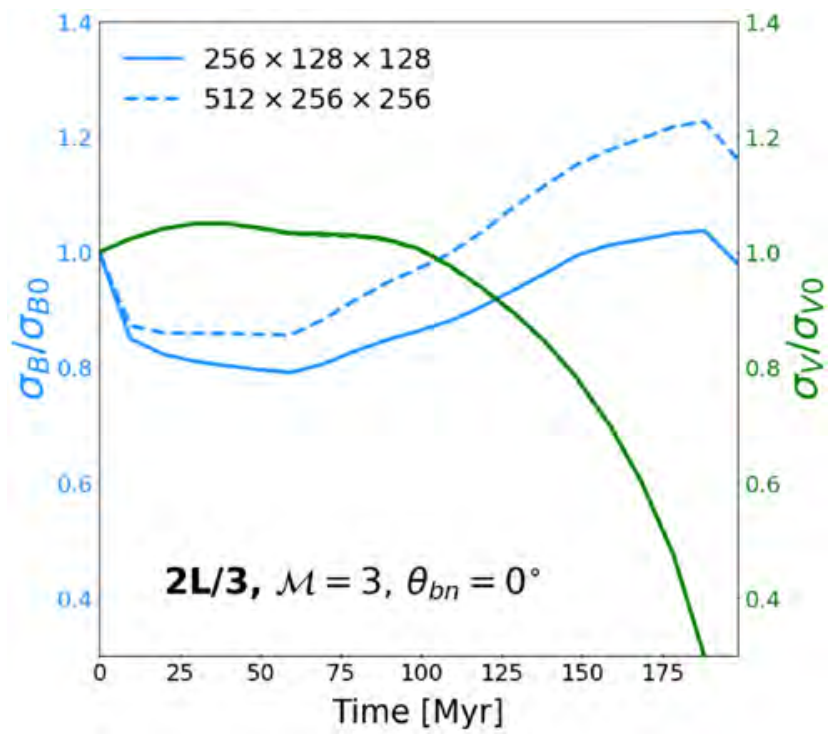

Figure C1. Evolution of the standard deviation of the magnetic field (left green axis) and velocity field (right blue axis). The solid lines correspond to the lower resolution run $(256 \times 128 \times 128$ cells $)$ and the dashed lines correspond to the higher resolution run $(512 \times 256 \times 256$ cells $)$. 

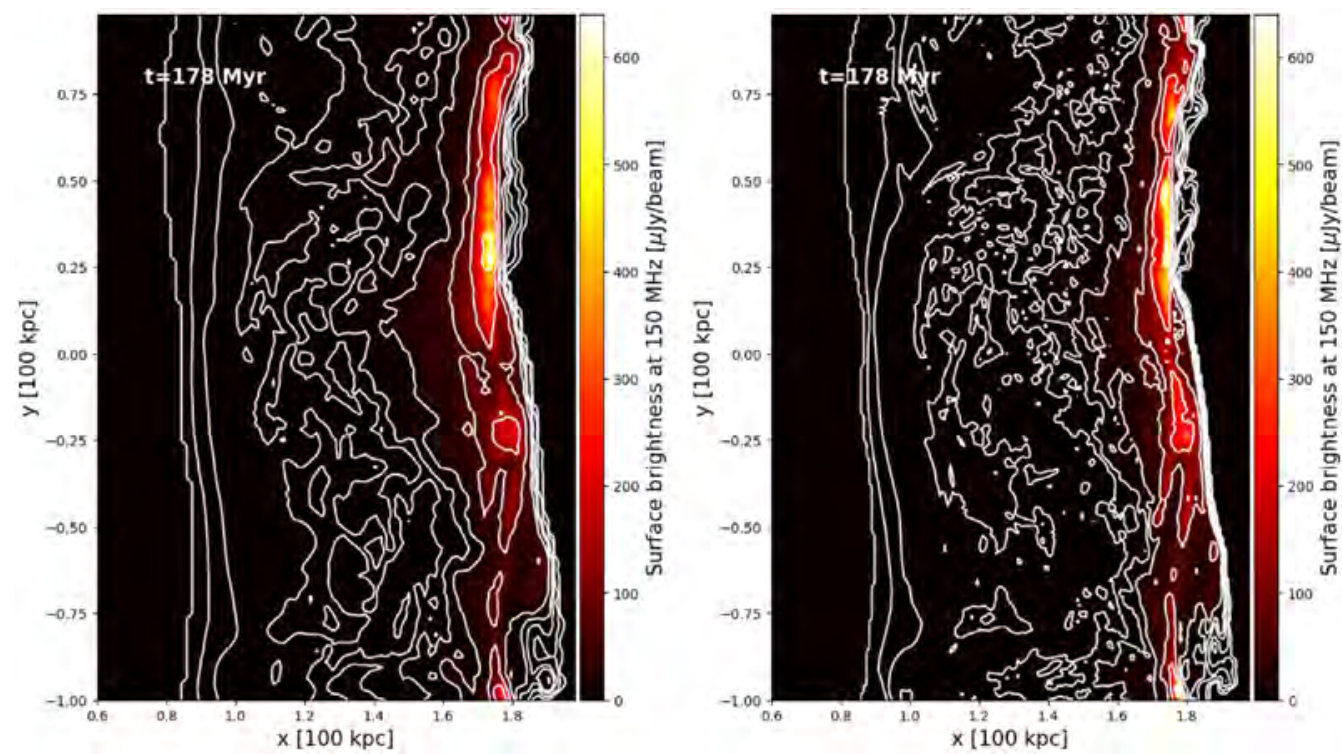

Figure C2. Surface brightness at $150 \mathrm{MHz}$ for the run $2 L / 3, \mathcal{M}=3$, and $\theta_{\mathrm{bn}}=90^{\circ}$. We considered a beam of $\theta^{2}=15$ arcsec $\times 15$ arcsec to get the surface brightness $\left(\theta^{2} I_{\nu}\right)$ in units of $\mu \mathrm{Jy}_{\text {beam }}{ }^{-1}$. No smoothing is considered here. Left-hand panel: $256 \times 128 \times 128$ cells simulation. Right-hand panel: $512 \times 256 \times$ 256 cells simulation.

ourselves to show the run $2 L / 3, \mathcal{M}=3$, and $\theta_{\text {bn }}=90^{\circ}$. In Fig. C1, we show the evolution of the standard deviation of the magnetic and velocity field for the resolution used in this work, i.e. $256 \times 128$ $\times 128$ cells, and for the higher resolution. This result was shown and explained in detail in Section 4.1. This figure confirms that the velocity field (and also the density and temperature field) dynamics is largely unaffected by a higher resolution. In fact, it is only the magnetic field fluctuations that are $\sim 20$ per cent enhanced. This means that our result on the non-correlation between magnetic and velocity fluctuations is even more accentuated at higher resolution. This is expected as we are increasing the effective Reynolds number of the simulation. An upper limit of the Reynolds number is given by

$\operatorname{Re}_{\max } \approx\left(\frac{l}{\Delta x}\right)^{4 / 3}$,

where $l$ is the maximum correlation scale in the flow and $\Delta x$ is the resolution. In this case, $l=2 L / 3 \approx 133.3 \mathrm{kpc}, \Delta x_{256}=1.56 \mathrm{kpc}$, and $\Delta x_{512}=0.78 \mathrm{kpc}$ would lead to an upper limit of the effective Reynolds number of $\sim 377$ and $\sim 950$ for the low- and high-resolution runs, respectively. A lower limit is given in contrast by

$\operatorname{Re}_{\min } \approx\left(\frac{l}{\varepsilon \Delta x}\right)^{4 / 3}$ where $\varepsilon$ is a factor depending on the diffusivity of the numerical method. For second-order finite difference/volume codes such as our case with the PLUTO code, one can assume $\varepsilon \approx 7$ (e.g. Kritsuk et al. 2011). This leads to a lower limit of the effective Reynolds number of $\sim 28$ and $\sim 71$ for the low- and high-resolution runs, respectively.

Finally, we show in Fig. C2 a comparison between surface brightness maps at $150 \mathrm{MHz}$ with both resolutions. As it can be observed, the broader features as well as the extent of the downstream are consistent in both resolutions, while the higher resolution run highlights smaller features $(\sim 1 \mathrm{kpc})$.

\section{APPENDIX D: SURFACE BRIGHTNESS ALONG THE $X$-AXIS}

We present the surface brightness maps at $150 \mathrm{MHz}$ for all our runs as viewed from the $x$-axis in Fig. D1. We want to point out that these maps were obtained by projecting the emissivity already used for this work along the $z$-axis. In order to compute self-consistently the surface brightness maps changing the LOS, one has to change the observing angle, $\theta_{\text {obs }}$, (see Section 3.3) and the vector $\hat{\mathbf{n}}_{\text {los }}$. This is turn shall be used for computing the integral and Doppler factor in the emissivity equation (see Section 3.2). The maximum value of surface brightness in each panel of Fig. D1, is lower than its correspondent panel in Fig. 10. This is because in this case, we end up summing up the contribution of the emissivity for a smaller region $(\sim 100 \mathrm{kpc})$. 

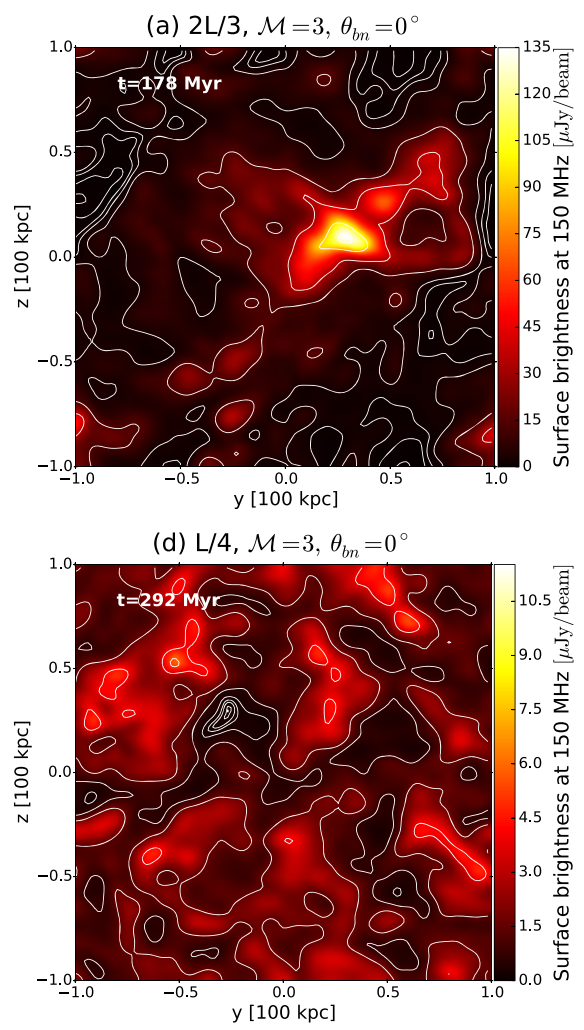

(b) $2 \mathrm{~L} / 3, \mathcal{M}=2, \theta_{b n}=0^{\circ}$

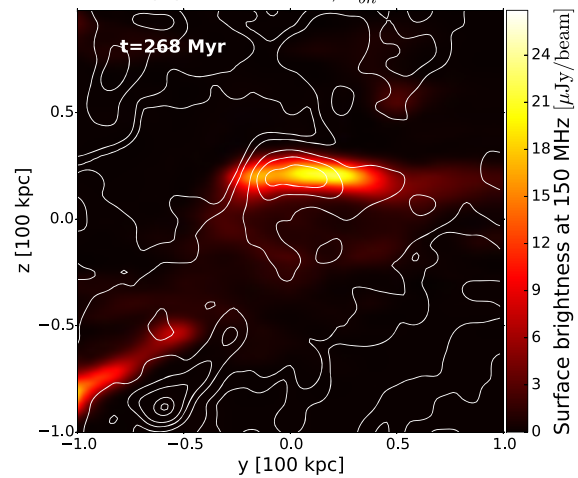

(e) $\mathrm{L} / 4, \mathcal{M}=2, \theta_{b n}=0^{\circ}$

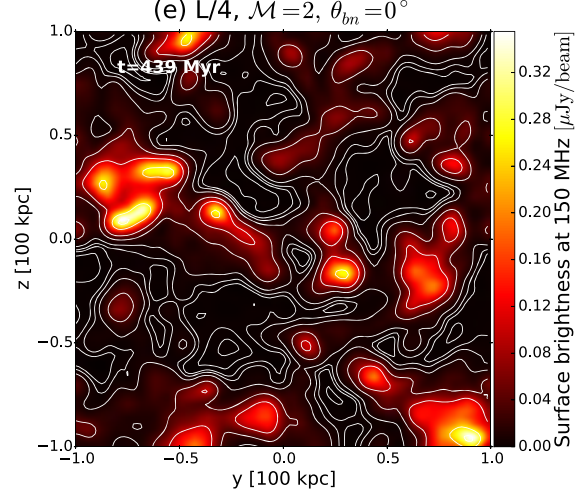

(c) $2 \mathrm{~L} / 3, \mathcal{M}=3, \theta_{b n}=90^{\circ}$

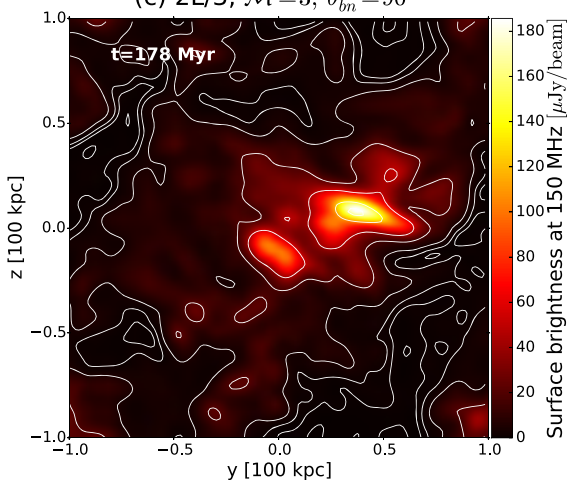

(f) $\mathrm{L} / 4, \mathcal{M}=3, \theta_{b n}=90^{\circ}$

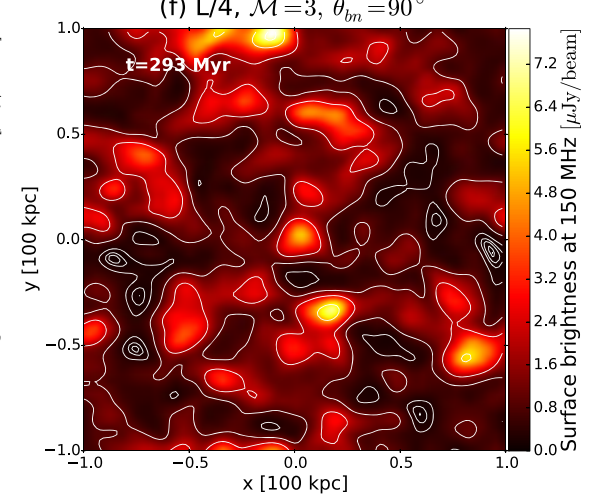

Figure D1. Surface brightness at $150 \mathrm{MHz}$ integrated along the $x$-axis (in correspondence to Fig. 10). We considered a beam of $\theta^{2}=15$ arcsec $\times 15$ arcsec to get the surface brightness $\left(\theta^{2} I_{\nu}\right)$ in units of $\mu \mathrm{Jy}$ beam $^{-1}$. We smoothed the maps with a Gaussian kernel with FWHM $=7.24 \mathrm{kpc}(\operatorname{assuming} z=0.023)$.

This paper has been typeset from a $\mathrm{T}_{\mathrm{E}} \mathrm{X} / \mathrm{LT} \mathrm{E} \mathrm{X}$ file prepared by the author. 